\title{
Quantitative analysis of headspace volatile compounds using comprehensive two-dimensional gas chromatography and their contribution to the aroma of Chardonnay wine
}

\author{
Juliane Elisa Welke a ${ }^{\text {, Mauro Zanus }}{ }^{\mathrm{b}}$, Marcelo Lazzarotto ${ }^{\mathrm{c}}$, Cláudia Alcaraz Zini ${ }^{\mathrm{d}, *}$ \\ a Instituto de Ciência e Tecnologia de Alimentos, Universidade Federal do Rio Grande do Sul, Avenida Bento Gonçalves, 9500, Porto Alegre, RS, Brazil \\ b Empresa Brasileira de Pesquisa Agropecuária, Embrapa Uva e Vinho, Rua Livramento, 515, Bento Gonçalves, RS, Brazil \\ c Empresa Brasileira de Pesquisa Agropecuária, Embrapa Florestas, Estrada da Ribeira, km 111, Colombo, PR, Brazil \\ ' Instituto de Química, Universidade Federal do Rio Grande do Sul, Avenida Bento Gonçalves, 9500, Porto Alegre, RS, Brazil
}

\section{A R T I C L E I N F O}

\section{Article history:}

Received 13 October 2013

Accepted 8 February 2014

Available online 15 February 2014

\section{Keywords:}

Comprehensive two-dimensional gas chroma-

tography

$\mathrm{GC} \times \mathrm{GC}$

Wine flavor

Quantification

SPME

Chardonnay

Relative odor contribution

ROC

\begin{abstract}
A B S T R A C T
The quantitative determination of volatile compounds of Chardonnay wines using HS-SPME-GC $\times$ GC/TOFMS along with the determination of odor activity value (OAV) and relative odor contribution (ROC) of volatiles are reported for the first time. The use of GC $\times$ GC/TOFMS for the analysis of Chardonnay wine of Serra Gaucha resulted in the tentative identification of 243 compounds, showing the superior performance of this analytical technique for this specific varietal wine, considering that the number of compounds usually separated by 1D-GC for this type of wine is lower. Furthermore, 42 compounds co-eluted in the first dimension and 34 of them were separated in the second dimension, while the others were resolved by spectral deconvolution (8), which indicates that the conventional 1D-GC/MS may result in misleading results. The calculation of OAV and ROC allowed the determination of the volatile compounds that presented the greater contribution to wine aroma. Ethyl octanoate, ethyl hexanoate, ethyl butanoate, and beta-damascenone showed the highest OAV and ROC values, although other 43 compounds showed also potential to contribute to wine aroma. Figures of merit of the developed method were: accuracies from 92.4 to $102.6 \%$, repeatability from $1.2 \%$ to $13.4 \%$, LOD from $0.001 \mu \mathrm{g} \mathrm{L}{ }^{-1}$ (ethyl isovalerate and hexanoic acid) to $2.554 \mu \mathrm{g} \mathrm{L}{ }^{-1}$ (ethyl 3-hydroxybutanoate), LOQ from $0.003 \mu \mathrm{g} \mathrm{L}{ }^{-1}$ (ethyl isovalerate and hexanoic acid) to $7.582 \mu \mathrm{g} \mathrm{L}^{-1}$ (ethyl 3-hydroxybutanoate).
\end{abstract}

(c) 2014 Elsevier Ltd. All rights reserved.

\section{Introduction}

Wine aroma is one of the most important factors that influence perceived wine quality and consumer acceptance (Bakker \& Clarke, 2011; Martínez-Pinilla, Guadalupe, Ayestarán, Pérez-Magariño, \& Ortega-Heras, 2013). Volatile compounds play a significant role to wine aroma and the presence, absence or different proportions of volatile compounds can be greatly influenced by both viticultural (climate, soil, cultivar, grape-growing practices) and enological (condition of grapes, fermentation, postfermentation treatments) factors (Jackson, 2008; Malherbe, Menichelli, du Toit, Tredoux, Muller, Næs, et al., 2013).

Hundreds of volatile compounds have been identified in wines (Robinson, Boss, Heymann, Solomon, \& Trengove, 2011; Weldegergis, de Villiers, McNeish, Seethapathy, Mostafa, Gorecki, et al., 2011; Welke, Manfroi, Zanus, Lazarotto, \& Zini, 2012). However, not all compounds present in wine contribute to aroma (Garcia-Carpintero, Sanchez-Palomo, Gallego, \& Gonzalez-Viñas, 2011; Li, Tao, Wang, \& Zhang, 2008); Vilanova, Genisheva, Masa, \& Oliveira, 2010). The

\footnotetext{
* Corresponding author. Tel.: + 55513308 72 17; fax: + 555133370442

E-mail address: cazini@iq.ufrgs.br (C. Alcaraz Zini).
}

influence of a volatile compound to the final aroma depends on its concentration in wine and on the perception threshold of this specific compound. The threshold of olfactory perception is defined as the lowest concentration capable of producing an olfactory sensation and that can be detected by human nose for at least $50 \%$ of the judges of a panel of sensory evaluation (Guth, 1997).

The determination of odor descriptors has been done by gas chromatography-olfactometry (GC-O), which uses the human nose as detector. The olfactive evaluation of the compounds by GC-O is performed after their separation in a chromatographic column, when their odor descriptors are perceived and registered, e.g., floral, fruity, green, solvent, plastic, toasted and others (Capone, Tufariello, \& Siciliano, 2013; Ferreira, Lopez, \& Cacho, 2000; Garcia-Carpintero, Sanchez-Palomo, Gallego, \& Gonzalez-Viñas, 2011; Jiang \& Zhang, 2010; Juan, Cacho, Ferreira, \& Escudero, 2012; Li et al., 2008; Peinado, Moreno, Muñoz, Medina, \& Moreno, 2004; Vilanova, Genisheva, Masa, \& Oliveira, 2010).

The determination of odor activity value (OAV) has also been extensively used to estimate the sensory contribution of aromatic compounds to the overall aroma of wine and it is usually performed in conjunction with 1D-GC/MS (Capone et al., 2013; Ferreira et al., 2000; Guth, 1997; Jiang \& Zhang, 2010; Juan et al., 2012). OAV is obtained from the 
ratio between the concentration of an individual compound and its perception threshold. A volatile compound contributes to aroma when its concentration in wine is above the perception threshold, therefore, odorants with OAV > 1 can be perceived (Guth, 1997). In addition to the use of OAV, which represents a quantitative approach of the contribution of volatile compounds to aroma, a qualitative evaluation can be done based on odor descriptors of each component. The relative odor contribution (ROC) represents the percentage of contribution of a particular aroma compound and it is the ratio of OAV percentage of each individual compound and the sum of the OAV of compounds that showed OAV $>1$ (Capone et al., 2013; Ferreira et al., 2000; Guth, 1997; Jiang \& Zhang, 2010; Juan et al., 2012; Li et al., 2008; Peinado et al., 2004). Capone et al. (2013) have identified 51 volatile compounds in Negroamaro red wines, and among them, only 18 components were perceived as active odorants ( OAV $>1)$. The compounds related to aroma were mainly alcohols, fatty acids and ethyl esters and the cited sensory descriptors were fruity, floral, fatty, pungent, nutty and caramelized notes.

The most suitable gas chromatography technique for untarget analysis of complex samples, such as wine, has been shown to be comprehensive two-dimensional gas chromatography $(\mathrm{GC} \times \mathrm{GC})$ (Welke and Zini, 2011). GC $\times$ GC has been quite often used in the determination of volatile compounds in different wine varieties, including Cabernet Franc (Ryona, Pan, \& Sacks, 2009), Madeira (Perestrelo, Barros, Câmara, \& Rocha, 2011), Cabernet Sauvignon (Robinson, Boss, Heymann, Solomon \& Trengove, 2011), Pinotage (Weldegergis et al., 2011), Muscat (Bordiga, Rinaldi, Locatelli, Piana, Travaglia, Coïsson et al., 2013) and Marsala (Dugo, Franchina, Scandinaro, Bonaccorsi, Cicero, Tranchida et al., 2014) wines. GC $\times$ GC offers superior separation capabilities afforded by high peak capacity, selectivity, structural chromatographic peak organization, and sensitivity enhancement in the same analysis time as in 1D-GC (Marriott \& Shellie, 2002). Specially valuable are the enrichment in aroma active trace compounds and the possibility of having two retention data, less co-elutions, and an organized distribution of wine volatile compounds in the 2D space, according to their physico-chemical characteristics, which helps the process of identification of unknown compounds. A former work of this research group on Merlot volatiles has been recently published, where the advantages of GC $\times$ GC/TOFMS have been highlighted through a detailed characterization of Merlot volatiles. Also, a preliminary approach to the use of multivariate analysis for discrimination of 24 wine samples according to grape variety has been presented (Welke, Manfroi, et al., 2012). Other previous works about qualitative determination of volatile compounds using GC $\times$ GC include the analysis of wines produced from different grape varieties (Welke, Manfroi, Zanus, Lazarotto, \& Zini, 2013) and the differentiation of base wines and the respective sparkling wines based on volatile profile (Welke, Zanus, Lazarotto, Pulgati, \& Zini, submitted for publication).

To the knowledge of the authors, there is no published work about the quantification of volatile compounds in Chardonnay wines using GC $\times$ GC/TOFMS combined with OAV/ROC approach in order to provide a fast overview regarding concentration of volatiles and odorant impact of the most important compounds of wine aroma. The aim of this study was to use the HS-SPME-GC $\times$ GC/TOFMS to quantify volatile compounds of wines produced with Chardonnay grapes in Serra Gaúcha region, Brazil.

\section{Material and methods}

\subsection{Samples, analytical reagents, and supplies}

Twelve wine samples ( $13 \%$ ethanol, v/v) produced with Chardonnay grape variety (Vitis vinífera L.) of 2009 vintage, produced in Serra Gaúcha region (latitude $29^{\circ} \mathrm{S}$, longitude $51^{\circ} \mathrm{W}$, altitude $600-800 \mathrm{~m}$ ) have been investigated. Each one of them was from different production batches. These samples were provided by the Empresa Brasileira de Pesquisa Agropecuária Uva e Vinho (EMBRAPA) in sealed $750 \mathrm{~mL}$ bottles and were chosen as the best wine samples in the National Evaluation of Wines of 2010, an event promoted by the Brazilian Association of Enology.

Standard compounds ethyl acetate, ethyl butanoate, ethyl propanoate, ethyl 2-methylpropanoate (ethyl isobutanoate), ethyl 2-methylbutanoate (ethyl isovalerate), ethyl hexanoate, ethyl 2-hydroxypropanoate (ethyl lactate), ethyl octanoate, ethyl decanoate, diethyl butanedioate (diethyl succinate), ethyl 2-hydroxybutanoate, 3-methylbutyl acetate (isoamyl acetate), ethyl 2-phenyl acetate, 1-propanol, 1-hexanol, 2-phenylethanol, hexanoic acid, octanoic acid, decanoic acid, dodecanoic acid, 4-terpineol, eugenol, and 3-octanol were purchased from Aldrich (Steinheim, Germany). These compounds were chosen considering their importance to flavor characteristics according to scientific reports (Jaffré, Valentin, Meunier, Siliani, Bertuccioli and Le Fur, 2011; Jiang \& Zhang, 2010; Li et al., 2008; Vilanova et al., 2010). Individual stock solutions of each compound ( $10 \mathrm{mg} \mathrm{L}^{-1}$ ) were prepared in bidistilled ethanol purchased from Nuclear (São Paulo, Brazil). Ethanol 10\% in MilliQ deionised water (purification system Millipore, Bedford, MA, USA) had been employed to prepare a solution of $6 \mathrm{~g} \mathrm{~L}^{-1}$ of $(+)$-tartaric acid (Synth, São Paulo, Brazil). The pH was adjusted to 3.5 with sodium hydroxide (Nuclear, São Paulo, Brazil). Calibration curves for each one of the analytes had been made through the addition of the 22 standard compounds to the model wine solution.

The SPME fiber (50/30 divinylbenzenecarboxen-polydimethylsiloxane (DVB/CAR/PDMS) StableFlex) was purchased from Supelco (Bellefonte, PA, USA) and was conditioned according to the manufacturer's recommendations prior to its first use. Sodium chloride $(\mathrm{NaCl})$ of analytical grade, purchased from Nuclear was oven dried at $110{ }^{\circ} \mathrm{C}$ overnight before use. Twenty microliter headspace vials with magnetic screw caps sealed with silicone septa had been bought from Supelco (Bellefonte, PA, USA).

\subsection{Instrumentation}

Extraction of volatile compounds from the headspace of the vials containing samples has been performed with a CTC CombiPAL autosampler (CTC Analytics, Zwingen, Switzerland) with an agitator and SPME fiber. The GC $\times$ GC system consisted of an Agilent 6890N (Agilent Technologies, Palo Alto, CA, USA) equipped with a Pegasus IV time-of-flight mass spectrometer (Leco Corporation, St. Joseph, MI, USA). A high polarity column (100\% polyethylene glycol; $30 \mathrm{~m} \times$ $0.25 \mathrm{~mm} \times 0.25 \mu \mathrm{m}$, J\&W Scientific Inc., Folsom, CA, USA) was used as first-dimension $\left({ }^{1} \mathrm{D}\right)$ column, and a DB-17ms (DB17ms (50\%-phenyl)methylpolysiloxane; $1.70 \mathrm{~m} \times 0.18 \mathrm{~mm} \times 0.18 \mu \mathrm{m}$, J\&W Scientific Inc., Folsom, CA, USA) was employed as a second-dimension $\left({ }^{2} \mathrm{D}\right)$ column. The GC system (Agilent 6890N) was equipped with a secondary column oven and non-moving quadjet dual stage thermal modulator. During modulation, cold pulses were generated using dry nitrogen gas cooled by liquid nitrogen (Linde, Canoas, RS, Brazil), whereas heated dry air was used for hot pulses. The injector, transfer line and ion source temperature were at $250{ }^{\circ} \mathrm{C}$. Oven temperature program conditions were as follows: initial temperature of $35{ }^{\circ} \mathrm{C}$ for $5 \mathrm{~min}$, programmed at $3{ }^{\circ} \mathrm{C} \mathrm{min}{ }^{-1}$ to $250{ }^{\circ} \mathrm{C}$, where it remained for $5 \mathrm{~min}$. The secondary oven was kept $10^{\circ} \mathrm{C}$ above the primary oven throughout the chromatographic run. The modulator was offset by $+25^{\circ} \mathrm{C}$ in relation to primary oven. Helium (99.9999\% purity, White Martins, Porto Alegre, RS, Brazil) was used as carrier gas at a constant flow of $1 \mathrm{~mL} \mathrm{~min}^{-1}$. The MS parameters included electron ionization at $70 \mathrm{eV}$ with ion source temperature at $250{ }^{\circ} \mathrm{C}$, detector voltage of $-1750 \mathrm{~V}$, mass range of $45-450 \mathrm{~m} / \mathrm{z}$, and acquisition rate of 100 spectra $s^{-1}$.

\subsection{Conditions for the extraction of volatiles}

The SPME extraction was performed according to previous work: $1 \mathrm{~mL}$ of wine in $20 \mathrm{~mL}$ glass headspace vials, $30 \%$ of $\mathrm{NaCl}(\mathrm{m} / \mathrm{v})$, without sample agitation, extraction time of $45 \mathrm{~min}$ and extraction temperature 
of $45^{\circ} \mathrm{C}$ (Welke, Zanus, Lazarotto, Schmitt \& Zini, 2012). The wine samples (10 mL measured with a volumetric pipette), were spiked with $10 \mu \mathrm{L}$ of alcoholic solution of 3 -octanol at $1.25 \mathrm{mg} \mathrm{L}^{-1}$, used as internal standard. All samples were kept at $45^{\circ} \mathrm{C}$ for 10 min prior to extraction. The headspace was sampled using a $2 \mathrm{~cm}$ DVB/CAR/PDMS 50/30 $\mu \mathrm{m}$ fiber. The volatile and semi-volatile compounds were desorbed in the GC inlet at $250{ }^{\circ} \mathrm{C}$ for $5 \mathrm{~min}$ and the fiber was reconditioned for $5 \mathrm{~min}$ at $260{ }^{\circ} \mathrm{C}$ prior to each analysis. All samples were analyzed in triplicate.

\subsection{Data processing and identification of compounds}

LECO ChromaTOF version 4.22 software was used for all acquisition control, data processing and Fisher Ratio calculations. Automated peak find and spectral deconvolution with a baseline offset of 0.5 and signal to noise of three have been employed during data treatment.

Twenty-two compounds (listed in Section 2.1) were positively identified through comparison of retention time and mass spectra data of unknown compounds with those of authentic standards. Tentative identification of wine volatile compounds had been achieved by comparing experimental linear temperature programmed retention indices (LTPRI) with retention indices reported in the literature for 1D-GC and a description of this procedure has already been reported elsewhere (von Muhlen, Zini, Caramao, \& Marriott, 2008). Retention data of a series of $n$-alkanes (C9-C24), under the same experimental conditions employed for the chromatographic analysis of wine volatiles had been used for experimental LTPRI calculation. Mass spectrometric information of each chromatographic peak was compared to NIST mass spectra library version 2005 , considering a minimum similarity value of $80 \%$. Whenever a LTPRI was not found in the scientific literature in order to match with the experimentally determined LTPRI, only the chemical class of the wine volatile compound was assigned.

\subsection{Quantitative analysis}

Work solutions of the standard compounds were prepared in model wine and the range of concentrations of each one of the compounds is listed in Table 1. The HS-SPME extraction was performed in five replicates for each one of the sample and standard solutions. 3-Octanol was chosen as internal standard and $10 \mu \mathrm{L}$ of a $0.625 \mathrm{mg} \mathrm{L}^{-1}$ solution in bidistilled ethanol was added to each standard solution and sample.

The analytical parameters determined were: linearity, limits of detection (LOD) and quantification (LOQ), precision (repeatability and intermediate precision) and accuracy. These procedures were done according to International Conference on Harmonization (ICH) guidelines (ICH - International Conference on Harmonization of Technical Requirements for Registration of Pharmaceuticals for Human Use,

Table 1

Figures of merit of the HS-SPME-GC $\times$ GC/TOFMS method used for the determination of volatile compounds in Chardonnay wines.

\begin{tabular}{|c|c|c|c|c|c|c|c|}
\hline \# & Compound & Validation range $\left(\mu \mathrm{g}^{-1}\right)$ & Regression equation $^{a}$ & $r^{2}$ & $\operatorname{LOD}\left(\mu \mathrm{L} \mathrm{L}^{-1}\right)$ & $\operatorname{LOQ}\left(\mu \mathrm{g} \mathrm{L}^{-1}\right)$ & $\mathrm{LOQ}^{\mathrm{b}}\left(\mu \mathrm{g} \mathrm{L}^{-1}\right)$ \\
\hline 1 & Ethyl acetate & $9610-20,880$ & $\mathrm{y}=0.0057 \mathrm{x}+149.76$ & 0.992 & 0.432 & 1.224 & $1.52^{\mathrm{c}}$ \\
\hline 2 & Ethyl propanoate & $1200-20,000$ & $\mathrm{y}=0.0194 \mathrm{x}+2.6239$ & 0.992 & 0.004 & 0.148 & $4.1^{\mathrm{d}}$ \\
\hline 3 & Ethyl butanoate & $2500-25,000$ & $y=0.0145 x+6.8157$ & 0.994 & 0.034 & 0.102 & $\begin{array}{l}0.128^{f} \\
0.115^{d} \\
0.88^{e}\end{array}$ \\
\hline 4 & Ethyl 2-methyl propanoate (ethyl isobutanoate) & $12-168$ & $\mathrm{y}=0.0259 \mathrm{x}+0.7643$ & 0.990 & 0.005 & 0.017 & $\begin{array}{l}0.82^{\mathrm{d}} \\
1.5^{\mathrm{e}}\end{array}$ \\
\hline 5 & 3-Methyl butyl acetate (isoamyl acetate) & $18-490$ & $\mathrm{y}=0.0455 \mathrm{x}+1.3978$ & 0.981 & 0.009 & 0.029 & $\begin{array}{l}0.126^{f} \\
0.37^{d} \\
0.098^{e}\end{array}$ \\
\hline 6 & Ethyl 2-methylbutanoate (ethyl isovalerate) & $3.5-56$ & $\mathrm{y}=1055 \mathrm{x}+0.1067$ & 0.995 & 0.001 & 0.003 & $0.0041^{\mathrm{c}}$ \\
\hline 7 & Ethyl hexanoate & $58-660$ & $\mathrm{y}=0.0524 \mathrm{x}+31.081$ & 0.998 & 0.034 & 0.102 & $\begin{array}{l}0.123^{f} \\
0.0083^{d} \\
0.072^{e}\end{array}$ \\
\hline 8 & Ethyl 2-hydoxy propanoate (ethyl lactate) & $59,000-688,800$ & $\mathrm{y}=0.0007 \mathrm{x}-0.4042$ & 0.991 & 0.026 & 0.079 & $\begin{array}{l}0.096^{f} \\
9.542^{c}\end{array}$ \\
\hline 9 & Ethyl octanoate & $52,000-260,000$ & $y=0.5842 x+14.806$ & 0.990 & 0.004 & 0.011 & $\begin{array}{l}0.281^{\mathrm{f}} \\
0.0031^{\mathrm{d}} \\
0.010^{\mathrm{e}}\end{array}$ \\
\hline 10 & Ethyl decanoate & $180-7840$ & $y=0.3236 x-28.933$ & 0.997 & 0.007 & 0.019 & $\begin{array}{l}0.056^{\mathrm{f}} \\
0.0006^{\mathrm{d}} \\
0.023^{\mathrm{e}}\end{array}$ \\
\hline 11 & Ethyl 3-hydroxy-butanoate & $4500-72,700$ & $\mathrm{y}=0.0022 \mathrm{x}+0.0547$ & 0.994 & 2.554 & 7.582 & $\begin{array}{l}42^{\mathrm{f}} \\
371^{\mathrm{e}}\end{array}$ \\
\hline 12 & Diethyl succinate & $24.88-2300$ & $y=0.0032 x+1.7595$ & 0.998 & 0.009 & 0.028 & $\begin{array}{l}1.170^{f} \\
0.58^{e}\end{array}$ \\
\hline 13 & Ethyl 2-phenyl acetate & $14.3-460$ & $\mathrm{y}=0.0931 \mathrm{x}+0.3078$ & 0.988 & 0.002 & 0.007 & $\begin{array}{l}0.157^{\mathrm{f}} \\
0.024^{\mathrm{d}} \\
0.057^{\mathrm{e}}\end{array}$ \\
\hline 14 & 1-Propanol & 15-192 & $y=9.10^{-5} x+0.4967$ & 0.980 & 0.048 & 0.112 & - \\
\hline 15 & 1-Hexanol & $51-9340$ & $\mathrm{y}=0.0441 \mathrm{x}-2.5073$ & 0.983 & 0.003 & 0.010 & $\begin{array}{l}0.348^{f} \\
0.28^{e}\end{array}$ \\
\hline 16 & 2-Phenylethanol & $1400-360,000$ & $y=0.0024 x+11.309$ & 0.990 & 0.094 & 0.285 & $\begin{array}{l}0.317^{f} \\
0.2228^{c}\end{array}$ \\
\hline 17 & 4-Terpineol & 5-65.3 & $\mathrm{y}=0.194+0.09$ & 0.977 & 0.297 & 0.965 & $3.1^{\mathrm{e}}$ \\
\hline 18 & Eugenol & $0.05-5.6$ & $\mathrm{y}=0.100+0.108$ & 0.972 & 0.002 & 0.007 & $0.056^{\mathrm{f}}$ \\
\hline 19 & Hexanoic acid & $8.73-78.6$ & $\mathrm{y}=0.0961 \mathrm{x}-0.7158$ & 0.979 & 0.001 & 0.003 & $0.048^{f}$ \\
\hline 20 & Octanoic acid & $2744-197,200$ & $\mathrm{y}=0.0011 \mathrm{x}+37.349$ & 0.948 & 0.112 & 0.340 & $0.679^{f}$ \\
\hline 21 & Decanoic acid & $20.2-1608$ & $\mathrm{y}=0.0667 \mathrm{x}-2.2706$ & 0.944 & 0.006 & 0.017 & $0.022^{\mathrm{f}}$ \\
\hline 22 & Dodecanoic acid & $5.48-49.6$ & $\mathrm{y}=0.2921 \mathrm{x}+29.027$ & 0.942 & 0.017 & 0.052 & - \\
\hline
\end{tabular}

a $\mathrm{x}$ designates concentration of volatile compounds $\left(\mu \mathrm{g} \mathrm{L}^{-1}\right)$ and y designates ratio of peak area of a volatile compound and peak area of 3-octanol.

b LOQ found in literature when HS-SPME-1D-GC/MS was used in the determination of the same volatile compound in the headspace of wine.

c Howard, Mike, and Riesen (2005).

d Antalick, Perello, and Revel (2010).

e Noguerol-Pato, González-Barreiro, Cancho-Grande, and Simal-Gándara (2009).

f Perestrelo, Fernandes, Albuquerque, Marques, and Câmara (2006) used liquid-liquid extraction with dichoromethane and 1D-GC/MS. 
Table 2

Tentatively identified compounds of Chardonnay wine headspace with their respective concentration, odor threshold, odor activity value (OAV) and odor descriptor.

\begin{tabular}{|c|c|c|c|c|c|c|c|c|c|c|}
\hline & Compound $^{\mathrm{a}}$ & CAS & $S^{b}$ & $\begin{array}{l}\text { LTPRI } \\
(\exp )^{\mathrm{c}}\end{array}$ & $\begin{array}{l}\text { LTPRI } \\
\text { (lit) }^{\mathrm{d}}\end{array}$ & $\begin{array}{l}\text { Conc. }^{\mathrm{e}} \\
\left(\mu \mathrm{g} \mathrm{L}^{-1}\right)\end{array}$ & $\begin{array}{l}\text { RSD } \\
(\%)^{f}\end{array}$ & $\begin{array}{l}\text { OTS } \\
\left(\mu \mathrm{g} \mathrm{L}^{-1}\right)^{\mathrm{h}}\end{array}$ & $\mathrm{OAV}^{\mathrm{g}}$ & Odor descriptor $^{\text {h }}$ \\
\hline \multicolumn{11}{|c|}{ Alcohols } \\
\hline 1 & 2-Propanol (isopropanol) ${ }^{\Phi}$ & $63-63-5$ & 909 & 926 & $938^{1}$ & 15.25 & 2.0 & $\mathrm{Nf}^{\mathrm{k}}$ & - & Pungent ${ }^{54}$ \\
\hline 2 & 1-Propanol ${ }^{\Phi}$ & $71-23-8$ & 934 & 1038 & $1036^{2}$ & 54.45 & 3.9 & $306,000^{49}$ & $<1$ & Alcohol, ripe fruit ${ }^{49}$ \\
\hline 3 & 2-Methyl-1-propanol (isobutyl alcohol) ${ }^{\Phi}$ & $75-65-0$ & 890 & 1091 & $1093^{3}$ & 110.67 & 6.7 & $75,000^{49}$ & $<1$ & Alcohol $^{49}$ \\
\hline 4 & 1-Penten-3-ol ${ }^{\Phi}$ & $616-25-1$ & 934 & 1102 & $1112^{4}$ & 60.09 & 4.8 & $400^{53, \mathrm{i}}$ & $<1$ & Green $^{53}$ \\
\hline 5 & $1-\mathrm{Butanol}^{\Phi}$ & $71-36-3$ & 903 & 1156 & $1165^{2}$ & 160.88 & 4.3 & $150,000^{49}$ & $<1$ & Medicinal $^{49}$ \\
\hline 6 & 3-Methyl-1-butanol (isoamyl alcohol) ${ }^{\Phi}$ & $137-32-6$ & 940 & 1208 & $1209^{3}$ & 65.72 & 8.3 & $60,000^{49}$ & $<1$ & Solvent ${ }^{49}$ \\
\hline 7 & 1 -Pentanol $(\mathbf{1})^{\Phi}$ & $71-41-0$ & 913 & 1245 & $1233^{4}$ & 24.45 & 6.4 & $64,000^{32}$ & $<1$ & Almond, synthetic, balsamic ${ }^{32}$ \\
\hline 8 & 3-Methyl-3-buten-1-ol ${ }^{\Phi}$ & $763-32-6$ & 980 & 1256 & $1254^{5}$ & 1200.89 & 7.5 & $600^{51}$ & 2.0 & Sweet fruity ${ }^{51}$ \\
\hline 9 & 2-Heptanol (18) ${ }^{\Psi}$ & $543-49-7$ & 934 & 1315 & $1316^{5}$ & 950.21 & 4.1 & $200^{51}$ & 4.7 & Lemon, orange, copper ${ }^{51}$ \\
\hline 10 & $(E)$-2-Penten-1-ol ${ }^{\Psi}$ & 20273-24-9 & 850 & 1326 & $1337^{55}$ & 60.87 & 4.2 & $89.2^{55, \mathrm{i}}$ & $<1$ & Green, plastic ${ }^{55}$ \\
\hline 11 & 4-Methyl-1-pentanol ${ }^{\Psi}$ & $626-89-1$ & 980 & 1333 & $1330^{2}$ & 6740.18 & 9.5 & $50,000^{32}$ & $<1$ & Almond, toasted ${ }^{32}$ \\
\hline 12 & 3-Methyl-1-pentanol ${ }^{\Psi}$ & $589-35-5$ & 929 & 1344 & $1343^{2}$ & 9020.90 & 6.4 & $50,000^{32}$ & $<1$ & Vinous, herbaceous, cacao 32 \\
\hline 13 & 1-Hepten-3-ol (3) ${ }^{\Psi}$ & $49-3852-7$ & 908 & 1350 & $1355^{6}$ & 95.96 & 8.5 & $\mathrm{Nf}$ & - & $\mathrm{Nf}$ \\
\hline 14 & 1-Hexanol ${ }^{\Psi}$ & $111-27-3$ & 902 & 1365 & $1357^{5}$ & 54.15 & 6.5 & $110^{49}$ & $<1$ & Herbaceous, grass, woody ${ }^{49}$ \\
\hline 15 & 3-Methyl-4-penten-1-ol ${ }^{\Psi}$ & $763-89-3$ & 887 & 1387 & $1390^{7}$ & 690.55 & 8.2 & $\mathrm{Nf}$ & - & $\mathrm{Nf}$ \\
\hline 16 & Cyclohexanol (19) $)^{\Omega}$ & $108-93-0$ & 807 & 1400 & $1407^{28}$ & 140.12 & 3.4 & $300^{52}$ & $<1$ & Camphor like odor, pungent ${ }^{52}$ \\
\hline 17 & 3-Ethoxy-1-propanol (4) ${ }^{\Psi}$ & $111-35-3$ & 935 & 1405 & $1409^{2}$ & 170.15 & 9.3 & $100^{51}$ & 1.7 & Fruity $^{52}$ \\
\hline 18 & (Z) 3-Hexen-1-ol (5) ${ }^{\Psi}$ & $928-96-1$ & 894 & 1409 & $1415^{2}$ & 880.99 & 6.9 & $1000^{49}$ & $<1$ & Green, bitter, fatty ${ }^{49}$ \\
\hline 19 & Alcohol $^{\Psi}$ & - & 810 & 1413 & $\mathrm{Nf}$ & 53.34 & 4.3 & - & - & - \\
\hline 20 & $(Z)-2$-Hexen-1-ol $(\mathbf{6})^{\Psi}$ & $928-95-0$ & 965 & 1425 & $1419^{4}$ & 70.32 & 3.1 & $400^{51}$ & $<1$ & Green grass, herb ${ }^{51}$ \\
\hline 21 & 1-Octen-3-ol ${ }^{\Psi}$ & $3391-86-4$ & 971 & 1439 & $1444^{59}$ & 0.97 & 2.4 & $1^{60, \mathrm{i}}$ & $<1$ & Chestnut flower, mushroomy ${ }^{59}$ \\
\hline 22 & 6-Methyl-5-hepten-2-ol (8) ${ }^{\Psi}$ & $110-93-0$ & 852 & 1450 & $1462^{6}$ & 60.59 & 1.4 & $2000^{53, \mathrm{i}}$ & $<1$ & $\mathrm{Nf}$ \\
\hline 23 & 2-Ethyl-1-hexanol ${ }^{\Psi}$ & $104-76-7$ & 899 & 1478 & $1474^{8}$ & 12150.1 & 7.3 & $8000^{51}$ & 1.5 & Floral $^{41}$, sweet fruity ${ }^{51}$ \\
\hline 24 & 2-Butyl-1-octanol ${ }^{\Psi}$ & $3913-02-8$ & 939 & 1523 & $1528^{9}$ & 1.32 & 6.4 & $\mathrm{Nf}$ & - & $\mathrm{Nf}$ \\
\hline 25 & 2-Nonanol ${ }^{\Psi}$ & $628-99-9$ & 955 & 1526 & $1530^{5}$ & 89.23 & 9.1 & $58^{53, \mathrm{i}}$ & 1.5 & Fruity, green ${ }^{53}$ \\
\hline 26 & 3-Ethyl-4-methylpentanol ${ }^{\Psi}$ & $100431-87-6$ & 939 & 1534 & $1531^{2}$ & 9.17 & 2.9 & $\mathrm{Nf}$ & - & $\mathrm{Nf}$ \\
\hline 27 & 2-(2-Methoxypropoxy)-1-propanol ${ }^{\Psi}$ & $83730-60-3$ & 909 & 1541 & $1532^{10}$ & 15.07 & 4.7 & $\mathrm{Nf}$ & - & $\mathrm{Nf}$ \\
\hline 28 & 2-(2-Methoxy ethoxy)ethanol ${ }^{\Psi}$ & $111-77-3$ & 872 & 1568 & $1576^{11}$ & 2.05 & 5.5 & $\mathrm{Nf}$ & - & $\mathrm{Nf}$ \\
\hline 29 & 2-Undecen-1-ol ${ }^{\Psi}$ & 37617-03-1 & 907 & 1594 & $1590^{12}$ & 8.09 & 7.6 & $\mathrm{Nf}$ & - & $\mathrm{Nf}$ \\
\hline 30 & 2,3-Butanediol $(\mathbf{9})^{\Psi}$ & $513-89-3$ & 932 & 1600 & $1598^{2}$ & 23.58 & 8.3 & $150,000^{32}$ & $<1$ & Fruity $^{32}$ \\
\hline 31 & 1,2-Propanediol ${ }^{\Psi}$ & $57-55-6$ & 917 & 1608 & $1603^{13}$ & 10.18 & 2.3 & $\mathrm{Nf}$ & - & $\mathrm{Nf}$ \\
\hline 32 & 2,7-Dimethyl-1-octanol $(\mathbf{1 0})^{\Psi}$ & $15-250-22-3$ & 878 & 1618 & $1628^{10}$ & 44.02 & 5.3 & $\mathrm{Nf}$ & - & $\mathrm{Nf}$ \\
\hline 33 & 2-(Ethoxyethoxy)ethanol ${ }^{\Psi}$ & $111-90-0$ & 876 & 1637 & $1636^{11}$ & 11.02 & 1.2 & $\mathrm{Nf}$ & - & $\mathrm{Nf}$ \\
\hline 34 & $(E)-2-N o n e n-1-\mathrm{ol}^{\Psi}$ & $31502-14-4$ & 905 & 1690 & $1697^{12}$ & 0.95 & 8.4 & $\mathrm{Nf}$ & - & $\mathrm{Nf}$ \\
\hline 35 & 4-Butoxy-1-butanol ${ }^{\Psi}$ & $4161-24-4$ & 897 & 1706 & $1701^{11}$ & 0.89 & 3.4 & $\mathrm{Nf}$ & - & $\mathrm{Nf}$ \\
\hline 36 & 2-Undecanol ${ }^{\Psi}$ & $1653-30-1$ & 982 & 1741 & $1738^{53}$ & 98.40 & 8.4 & $41^{53, \mathrm{i}}$ & 2.4 & Fruity $^{53}$ \\
\hline 37 & $(E)-3-$ Decenol $^{\Psi}$ & $10339-60-3$ & 861 & 1757 & $1759^{6}$ & 10.04 & 6.7 & $\mathrm{Nf}$ & - & $\mathrm{Nf}$ \\
\hline 38 & 1-Decanol ${ }^{\Psi}$ & $112-30-1$ & 890 & 1778 & $1781^{2}$ & 29.89 & 2.9 & $400^{52}$ & $<1$ & Sweet, fatty ${ }^{3}$ \\
\hline 39 & $(Z)$-4-decen-2-ol ${ }^{\prime}$ & $57074-37-0$ & 859 & 1786 & $1794^{5}$ & 88.01 & 7.1 & $\mathrm{Nf}$ & - & $\mathrm{Nf}$ \\
\hline 40 & Alcohol $(\mathbf{2 0})^{\Psi}$ & - & 858 & 1805 & $\mathrm{Nf}$ & 10.43 & 6.7 & - & - & - \\
\hline 41 & Phenylmethanol (benzylalcohol) $)^{\Omega}$ & $100-51-6$ & 940 & 1875 & $1879^{5}$ & 12376.97 & 3.4 & $200,000^{32}$ & $<1$ & Sweet, fruity ${ }^{32}$ \\
\hline 42 & 3-Methoxy-2-butanol ${ }^{\Psi}$ & $53778-72-6$ & 941 & 1889 & $1903^{15}$ & 81.08 & 9.0 & $\mathrm{Nf}$ & - & $\mathrm{Nf}$ \\
\hline 43 & 2-Phenylethanol ${ }^{\Omega}$ & $60-12-8$ & 930 & 1947 & $1946^{3}$ & $356,679.48$ & 3.5 & $200,000^{49}$ & 1.8 & Rose, honey ${ }^{49}$ \\
\hline 44 & 2,6-Dimethyl-3,7-octadien-2,6-diol ${ }^{\Psi}$ & $13741-21-4$ & 858 & 1954 & $1951^{7}$ & 20.01 & 9.6 & $\mathrm{Nf}$ & - & $\mathrm{Nf}$ \\
\hline 45 & 1-Dodecanol ${ }^{\Psi}$ & $12-53-8$ & 928 & 1984 & $1981^{3}$ & 45.98 & 3.8 & $1000^{57}$ & $<1$ & $\begin{array}{l}\text { Unpleasant in higher concentration, } \\
\text { flowery in low concentration }\end{array}$ \\
\hline 46 & 1-Tridecanol $^{\Psi}$ & $112-70-9$ & 853 & 2066 & $2063^{16}$ & 10.06 & 9.3 & $\mathrm{Nf}$ & - & $\mathrm{Nf}$ \\
\hline 47 & 2-Phenoxyethanol (16) $)^{\Omega}$ & $122-99-6$ & 810 & 2119 & $2115^{39}$ & 170.49 & 4.0 & $\mathrm{Nf}$ & - & $\mathrm{Nf}$ \\
\hline 48 & Pentadecanol $^{\Psi}$ & $1653-34-5$ & 986 & 2200 & $2197^{17}$ & 9.07 & 9.2 & $\mathrm{Nf}$ & - & $\mathrm{Nf}$ \\
\hline 49 & 5-(2-Propynyloxy)-2-pentanol ${ }^{\Psi}$ & $55702-67-5$ & 876 & 2272 & $2273^{18}$ & 6.13 & 8.8 & $\mathrm{Nf}$ & - & $\mathrm{Nf}$ \\
\hline 50 & 2-Hexadecanol ${ }^{\Psi}$ & $14852-31-4$ & 912 & 2302 & $2312^{18}$ & 51.40 & 3.4 & $\mathrm{Nf}$ & - & $\mathrm{Nf}$ \\
\hline 51 & 1-Phenyl-1,2-propanediol ${ }^{\Omega}$ & $1855-09-9$ & 943 & $2465^{\mathrm{j}}$ & $2455^{19}$ & 144.15 & 5.6 & $\mathrm{Nf}$ & - & $\mathrm{Nf}$ \\
\hline 52 & 1-Heptadecanol ${ }^{\Psi}$ & $1454-85-9$ & 982 & $2461^{\mathrm{j}}$ & $2475^{20}$ & 52.45 & 3.4 & $\mathrm{Nf}$ & - & $\mathrm{Nf}$ \\
\hline \multicolumn{11}{|c|}{ Acids } \\
\hline 53 & 4-Methyl-2-oxovaleric acid $§$ & $816-66-0$ & 876 & 1434 & $1421^{21}$ & 11.48 & 4.1 & $\mathrm{Nf}$ & - & $\mathrm{Nf}$ \\
\hline 54 & Acetic acid $\S$ & $64-19-7$ & 890 & 1457 & $1461^{3}$ & 5605.98 & 7.9 & $200000^{50}$ & $<1$ & Pungent, vinegar ${ }^{32}$ \\
\hline 55 & Propanoic acid ${ }^{\S}$ & $79-09-4$ & 872 & 1536 & $1525^{15}$ & 3400.56 & 6.5 & $8100^{32}$ & $<1$ & Pungent, rancid, soy ${ }^{32}$ \\
\hline 56 & 2-Methylpropanoic acid (isobutyric acid) ( $^{\S}$ & $79-31-2$ & 863 & 1605 & $1618^{2}$ & 8559.40 & 3.6 & $200,000^{50}$ & $<1$ & Cheese $^{26}$ \\
\hline 57 & Acid $^{\S}$ & - & 876 & 1584 & $\mathrm{Nf}$ & 7.94 & 4.5 & - & - & - \\
\hline 58 & Butanoic acid $(\mathbf{1 2})^{\S}$ & $107-92-6$ & 930 & 1641 & $1637^{10}$ & 890.89 & 4.0 & $10,000^{50}$ & $<1$ & Rancid, cheese $\mathrm{e}^{32}$ \\
\hline 59 & $\mathrm{Acid}^{\S}$ & - & 859 & 1658 & $\mathrm{Nf}$ & 20.24 & 3.4 & - & - & - \\
\hline 60 & 3-Methyl butanoic acid (isovaleric acid) $(\mathbf{1 3})^{\S}$ & $503-74-2$ & 931 & 1664 & $1660^{22}$ & 20.87 & 7.7 & $3000^{50}$ & $<1$ & Rancid, acidic ${ }^{41}$ \\
\hline 61 & Pentanoic acid $\$$ & $109-52-4$ & 863 & 1750 & $1744^{10}$ & 86.76 & 3.9 & $3000^{60, \mathrm{i}}$ & $<1$ & Cheese $^{58}$ \\
\hline 62 & $(Z)$-2-Methyl-2-butenoic acid $(\mathbf{1 7})^{\S}$ & $80-59-1$ & 857 & 1839 & $1846^{59}$ & 32.48 & 6.7 & $\mathrm{Nf}$ & - & Spicy, pungent ${ }^{59}$ \\
\hline 63 & Hexanoic acid $\$$ & $142-62-1$ & 940 & 1876 & $1863^{2}$ & 49.59 & 7.3 & $3000^{49}$ & 1.5 & Cheese, fatty ${ }^{49}$ \\
\hline 64 & 2-Ethyl-hexanoic acid§ & $149-57-5$ & 979 & 1956 & $1950^{35}$ & 70.18 & 1.8 & $\mathrm{Nf}$ & - & $\mathrm{Nf}$ \\
\hline 65 & Heptanoic acid $\S$ & $111-14-8$ & 956 & 1974 & $1960^{10}$ & 20.34 & 9.2 & $\mathrm{Nf}$ & - & Sweaty, cheese $\mathrm{e}^{58}$ \\
\hline 66 & (E)-2-Hexenoic acid§ & $13419-69-7$ & 901 & 1980 & $1990^{7}$ & 12.01 & 4.7 & $\mathrm{Nf}$ & - & $\mathrm{Nf}$ \\
\hline 67 & Octanoic acid ${ }^{\star}$ & $124-07-2$ & 956 & 2086 & $2083^{2}$ & $160,320.82$ & 6.7 & $10,000^{49}$ & 16 & Fatty, rancid ${ }^{49}$ \\
\hline 68 & Nonanoic acid ${ }^{\star}$ & $112-05-0$ & 904 & 2090 & $2091^{23}$ & 1820.65 & 8.3 & $3000^{41}$ & & Fatty 41 \\
\hline 69 & 4-Methyl octanoic acid ${ }^{\star}$ & $54947-74-9$ & 963 & 2167 & $2173^{24}$ & 29.09 & 6.1 & $\mathrm{Nf}$ & - & $\mathrm{Nf}$ \\
\hline 70 & Decanoic acid $^{\infty}$ & $334-48-5$ & 942 & 2287 & $2296^{2}$ & 180.10 & 5.1 & $6^{49}$ & 3 & Fatty, rancid ${ }^{49}$ \\
\hline 71 & 9-Decenoic acid $^{\infty}$ & $14436-32-9$ & 917 & 2350 & $2348^{25}$ & 30.56 & 9.3 & $40^{52}$ & $<1$ & Waxy, fatty, soapy ${ }^{52}$ \\
\hline
\end{tabular}


Table 2 (continued)

\begin{tabular}{|c|c|c|c|c|c|c|c|c|c|c|}
\hline & Compound $^{\mathrm{a}}$ & CAS & $S^{b}$ & $\begin{array}{l}\text { LTPRI } \\
(\exp )^{c}\end{array}$ & 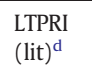 & $\begin{array}{l}\text { Conc. }^{\mathrm{e}} \\
\left(\mu \mathrm{g} \mathrm{L}^{-1}\right)\end{array}$ & $\begin{array}{l}\text { RSD } \\
(\%)^{f}\end{array}$ & $\begin{array}{l}\text { OTS } \\
\left(\mu \mathrm{g} \mathrm{L}^{-1}\right)^{\mathrm{h}}\end{array}$ & $O A V^{g}$ & Odor descriptor $^{\mathrm{h}}$ \\
\hline \multicolumn{11}{|l|}{ Acids } \\
\hline 72 & Undecanoic acid ${ }^{\infty}$ & $112-37-8$ & 901 & 2369 & $2365^{22}$ & 42.91 & 5.3 & $\mathrm{Nf}$ & - & Oily ${ }^{30}$ \\
\hline 73 & Dodecanoic acid* & $143-07-7$ & 856 & $2508^{\mathrm{j}}$ & $2517^{2}$ & 22.47 & 6.1 & $1000^{57}$ & $<1$ & Dry, metallic ${ }^{57}$ \\
\hline 74 & 2-Phenylacetic acid* & $103-82-2$ & 907 & $2580^{\mathrm{j}}$ & $2585^{26}$ & 43.92 & 8.2 & $1000^{52}$ & $<1$ & Honey, pollen, roses ${ }^{26}$ \\
\hline 75 & 3-Phenylpropanoic acid* & $501-52-0$ & 865 & $2613^{\mathrm{j}}$ & $2603^{8}$ & 49.23 & 4.5 & $\mathrm{Nf}$ & - & Floral, fruity ${ }^{8}$ \\
\hline 76 & Tetradecanoic acid** & $544-63-8$ & 893 & $2855^{\mathrm{j}}$ & $2847^{2}$ & 65.62 & 5.9 & $10,000^{62, \mathrm{i}}$ & $<1$ & $\mathrm{Nf}$ \\
\hline 77 & Hexadecanoic acid $*$ & $57-10-3$ & 845 & $2870^{j}$ & $2862^{23}$ & 46.61 & 1.0 & $10,000^{62, \mathrm{i}}$ & $<1$ & $\mathrm{Nf}$ \\
\hline 78 & - & - & 862 & $2889^{j}$ & $\mathrm{Nf}$ & 40.19 & 2.7 & $\mathrm{Nf}$ & - & $\mathrm{Nf}$ \\
\hline \multicolumn{11}{|c|}{ Aldehydes } \\
\hline 79 & Acetaldehyde ${ }^{\Psi}$ & $75-07-0$ & 871 & $700^{j}$ & $692^{3}$ & 78.65 & 5.1 & $500^{50}$ & $<1$ & Pungent $^{3}$ \\
\hline 80 & 2-Propenal (acrolein) ${ }^{\Psi}$ & $107-02-8$ & 831 & $858^{\mathrm{j}}$ & $846^{28}$ & 79.76 & 4.3 & $\mathrm{Nf}$ & - & $\mathrm{Nf}$ \\
\hline 81 & 3-Methyl-1-butanal ${ }^{\Psi}$ & $590-86-3$ & 901 & 910 & $915^{8}$ & 0.01 & 8.9 & $0.35^{53, i}$ & $<1$ & Green, malt ${ }^{41}$ \\
\hline 82 & 2-Butenal ${ }^{\Psi}$ & $4170-30-3$ & 950 & 1050 & $1041^{28}$ & 0.11 & 3.2 & $0.3^{55, \mathrm{i}}$ & $<1$ & Pungent $t^{55}$ \\
\hline 83 & Hexanal $^{\Psi}$ & $66-25-1$ & 943 & 1107 & $1097^{25}$ & 9.71 & 3.4 & $4.5^{53, \mathrm{i}}$ & 2.1 & Sweet, orange ${ }^{3}$ \\
\hline 84 & 2,3-Dimethylpentanal $(\mathbf{2})^{\Psi}$ & $32749-94-3$ & 932 & 1260 & $1258^{18}$ & 5.12 & 7.5 & $\mathrm{Nf}$ & - & $\mathrm{Nf}$ \\
\hline 85 & Octanal $^{\Psi}$ & $124-13-0$ & 941 & 1292 & $1280^{10}$ & 8.51 & 5.4 & $15^{26}$ & $<1$ & Fatty ${ }^{3}$ \\
\hline 86 & Nonanal $(\mathbf{1 9})^{\Psi}$ & $124-19-6$ & 960 & 1399 & $1408^{3}$ & 42.09 & 5.6 & $15^{26}$ & 2.8 & Citrusy, floral ${ }^{3}$ \\
\hline 87 & Decanal $^{\Psi}$ & $112-31-2$ & 981 & 1507 & $1515^{3}$ & 0.17 & 9.7 & $1^{26}$ & $<1$ & Grassy, orange skin-like ${ }^{41}$ \\
\hline 88 & 4-Ethylbenzaldehyde ${ }^{\Psi}$ & $4748-78-1$ & 943 & 1519 & $1521^{15}$ & 48.86 & 3.4 & $\mathrm{Nf}$ & - & Fruity, anised ${ }^{29}$ \\
\hline 89 & Benzaldehyde ${ }^{\Psi}$ & $100-52-7$ & 967 & 1516 & $1529^{28}$ & 764.85 & 5.9 & $2000^{49}$ & $<1$ & Almond ${ }^{49}$ \\
\hline 90 & Undecanal $^{\Psi}$ & $112-44-7$ & 984 & 1630 & $1622^{3}$ & 3.56 & 3.8 & $5^{47, i}$ & $<1$ & Waxy, floral $^{3}$ \\
\hline 91 & 2-Phenylacetaldehyde (benzeneacetaldehyde) ${ }^{\Psi}$ & $122-78-1$ & 896 & 1638 & $1631^{21}$ & 1.67 & 3.7 & $1^{26}$ & 1.7 & Flowery, rose $\mathrm{e}^{26}$ \\
\hline 92 & 4-Methylbenzaldehyde $(\mathbf{1 3})^{\Psi}$ & $104-87-0$ & 987 & 1665 & $1654^{29}$ & 56.12 & 8.6 & $\mathrm{Nf}$ & - & Almond $^{29}$ \\
\hline 93 & Dodecanal $^{\Psi}$ & $112-54-9$ & 864 & 1733 & $1729^{3}$ & 1.45 & 5.0 & $2^{47, \mathrm{i}}$ & $<1$ & Floral, waxy ${ }^{3}$ \\
\hline 94 & Tridecanal $^{\Psi}$ & $10486-19-8$ & 852 & 1820 & $1824^{30}$ & 30.67 & 7.3 & $\mathrm{Nf}$ & - & Sweet, fruity ${ }^{30}$ \\
\hline 95 & Tetradecanal $^{\Psi}$ & $124-25-4$ & 816 & 1893 & $1888^{14}$ & 4.86 & 5.1 & $6^{29, \mathrm{i}}$ & $<1$ & Floral, waxy ${ }^{29}$ \\
\hline 96 & 3-Phenyl-2-propenal (Cinnamaldehyde) ${ }^{\Psi}$ & $104-55-2$ & 843 & 1978 & $1970^{31}$ & 20.97 & 7.0 & $\mathrm{Nf}$ & - & $\mathrm{Nf}$ \\
\hline 97 & 4-Methoxybenzaldehyde ( $p$-anisaldehyde $)^{\Psi}$ & $123-11-5$ & 874 & 1984 & $1986^{39}$ & 75.80 & 3.8 & $\mathrm{Nf}$ & - & $\mathrm{Nf}$ \\
\hline 98 & Pentadecanal $^{\Psi}$ & $2765-11-9$ & 921 & 2034 & $2024^{27}$ & 64.02 & 8.2 & $\mathrm{Nf}$ & - & $\mathrm{Nf}$ \\
\hline 99 & Hexadecanal $^{\Psi}$ & $629-80-1$ & 963 & 2091 & $2094^{14}$ & 56.98 & 4.5 & $75^{62, \mathrm{i}}$ & $<1$ & $\mathrm{Nf}$ \\
\hline 100 & 4-Hydroxybenzaldehyde ${ }^{\Psi}$ & $123-08-0$ & 985 & 3070 & $3072^{32}$ & 49.02 & 8.4 & $\mathrm{Nf}$ & - & $\mathrm{Nf}$ \\
\hline \multicolumn{11}{|c|}{ Esters } \\
\hline 101 & Ethyl acetate $^{\mathrm{n}}$ & $141-78-6$ & 951 & 890 & $885^{2}$ & $15,608.09$ & 6.0 & $12,000^{49}$ & 1.3 & Pineapple, fruity, solvent ${ }^{49}$ \\
\hline 102 & Ethyl propanoate $^{o}$ & & 930 & 948 & $946^{3}$ & $10,450.44$ & 7.4 & $5500^{65}$ & 1.9 & Sweet, fruity ${ }^{3}$ \\
\hline 103 & Ethyl 2-methylpropanoate (ethyl isobutanoate) ${ }^{\circ}$ & $97-62-1$ & 896 & 968 & $965^{3}$ & 30.08 & 4.8 & $15^{65}$ & 2.0 & Sweet, fruity ${ }^{3}$ \\
\hline 104 & Ethyl butanoate $^{\mathrm{p}}$ & $105-54-4$ & 982 & 1028 & $1022^{22}$ & $15,189.87$ & 5.4 & $400^{49}$ & 38.0 & Strawberry, apple ${ }^{49}$ \\
\hline 105 & Ethyl 2-methylbutanoate (ethyl isovalerate) ${ }^{\mathrm{s}}$ & $7452-79-1$ & 971 & 1040 & $1035^{22}$ & 5.31 & 4.3 & $1^{50}$ & 5.3 & Apple, sweet $^{3}$ \\
\hline 106 & Ethyl pentanoate & $539-82-2$ & 934 & 1132 & $1142^{5}$ & 10.52 & 3.8 & $5^{62, \mathrm{i}}$ & 2.1 & Fruity $^{3}$, apple $^{8}$ \\
\hline 107 & Butyl ethanoate (Butyl acetate) $)^{\mathrm{q}}$ & $123-86-4$ & 925 & 1063 & $1074^{33}$ & $14,040.93$ & 8.2 & $1800^{49}$ & 7.8 & Fruity 49 \\
\hline 108 & 3-Methylbutyl acetate (isoamyl acetate) ${ }^{\mathrm{r}}$ & $123-92-2$ & 825 & 1129 & $1127^{34}$ & 1536.07 & 5.1 & $160^{49}$ & 9.6 & Banana, fruity, sweet ${ }^{49}$ \\
\hline 109 & Isobutyl 3-methylbutanoate ${ }^{\mathrm{r}}$ & $557-00-6$ & 863 & 1175 & $1175^{28}$ & 27.55 & 5.5 & $\mathrm{Nf}$ & - & $\mathrm{Nf}$ \\
\hline 110 & Butyl butanoate ${ }^{r}$ & $109-21-7$ & 841 & 1199 & $1212^{1}$ & 34.78 & 1.4 & $100^{61, \mathrm{i}}$ & $<1$ & $\mathrm{Nf}$ \\
\hline 111 & Ethyl hexanoate ${ }^{t}$ & $123-66-0$ & 836 & 1238 & $1244^{2}$ & 385.96 & 11.8 & $8^{49}$ & 48.2 & Fruity, green apple, brandy, wine-like 49 \\
\hline 112 & Ethyl 2-oxopropanoate $(\mathbf{1})^{\mathrm{t}}$ & $617-35-6$ & 973 & 1247 & $1242^{13}$ & 43.53 & 4.9 & $\mathrm{Nf}$ & - & $\mathrm{Nf}$ \\
\hline 113 & Hexyl acetate & $142-92-7$ & 919 & 1281 & $1270^{5}$ & 1072.39 & 4.6 & $670^{49}$ & 1.6 & Apple, cherry, pear, floral ${ }^{49}$ \\
\hline 114 & Ethyl heptanoate (18) ${ }^{\mathrm{t}}$ & $106-30-9$ & 927 & 1314 & $1317^{2}$ & 5.57 & 5.2 & $2.2^{61, \mathrm{i}}$ & 2.5 & Fruity $^{8}$ \\
\hline 115 & Propyl hexanoate $\mathrm{t}^{\mathrm{t}}$ & $626-77-7$ & 934 & 1322 & $1321^{33}$ & 124.75 & 3.2 & $\mathrm{Nf}$ & - & Fruity $^{8}$ \\
\hline 116 & Ethyl 2-hexenoate ${ }^{t}$ & $27829-72-7$ & 962 & 1335 & $1335^{5}$ & 167.25 & 4.2 & $\mathrm{Nf}$ & - & Fruity, pineapple ${ }^{53}$ \\
\hline 117 & Ethyl 2-hydroxypropanoate (ethyl lactate) ${ }^{\mathrm{u}}$ & $97-64-3$ & 921 & 1364 & $1363^{2}$ & 345127.41 & 5.7 & $150,000^{49}$ & 2.3 & Fruity, buttery ${ }^{49}$ \\
\hline 118 & Methyl octanoate ${ }^{\mathrm{v}}$ & $111-11-5$ & 804 & 1371 & $1378^{22}$ & 280.00 & 7.2 & $200^{63}$ & 1.4 & Fruity, citrus like ${ }^{63}$ \\
\hline 119 & Ethyl 2-hydroxy-3-methylbutanoate $(4)^{z}$ & 2441-06-7 & 856 & 1405 & $1399^{8}$ & 2589.78 & 4.6 & $1000^{51}$ & 2.6 & Pineapple, strawberry, tea, honey ${ }^{51}$ \\
\hline 120 & Ethyl 2-hydroxybutanoate $(\mathbf{5})^{z}$ & 52089-54-0 & 912 & 1409 & $1400^{8}$ & 24875.98 & 6.6 & $20,000^{65}$ & 1.2 & Fruity, floral ${ }^{8}$ \\
\hline 121 & Propyl heptanoate $(\mathbf{6})^{\mathrm{v}}$ & $7778-87-2$ & 941 & 1426 & $1425^{28}$ & 60.79 & 4.5 & $\mathrm{Nf}$ & - & $\mathrm{Nf}$ \\
\hline 122 & 2-Methylpropyl octanoate (isobutyl octanoate) ${ }^{\mathrm{v}}$ & $5461-06-3$ & 809 & 1545 & $1551^{5}$ & 88.74 & 7.3 & $0.8^{57}$ & $<1$ & $\mathrm{Nf}$ \\
\hline 123 & Hexyl 3-methylbutanoate (hexyl isovalerate) (7) & $10032-13-0$ & 869 & 1433 & $1425^{1}$ & 88.65 & 7.8 & $\mathrm{Nf}$ & - & $\mathrm{Nf}$ \\
\hline 124 & Ethyl 2-(1-ethoxyethoxy)propanoate ${ }^{v}$ & $37101-80-7$ & 874 & 1447 & $1442^{21}$ & 102.84 & 9.3 & $\mathrm{Nf}$ & - & $\mathrm{Nf}$ \\
\hline 125 & Ethyl octanoate $(7)^{\mathrm{v}}$ & $106-32-1$ & 988 & 1434 & $1446^{2}$ & 75450.87 & 6.3 & $580^{49}$ & 130 & Sweet, fruity, pear ${ }^{49}$ \\
\hline 126 & 3-Methylbutyl hexanoate (isopentyl hexanoate) ${ }^{\mathrm{v}}$ & 2198-61-0 & 941 & 1445 & $1450^{2}$ & 1251.22 & 2.8 & $1000^{57}$ & 1.2 & Sweet fruity ${ }^{3}$ \\
\hline 127 & Isobutyl 2-hydroxypropanoate ${ }^{z}$ & $73523-02-1$ & 988 & 1455 & $1455^{21}$ & 56.49 & 5.3 & $\mathrm{Nf}$ & - & $\mathrm{Nf}$ \\
\hline 128 & 3-Methyl octenoate & $69668-85-5$ & 976 & 1475 & $1483^{36}$ & 47.97 & 7.8 & $\mathrm{Nf}$ & - & $\mathrm{Nf}$ \\
\hline 129 & Ethyl 2,2-diethoxyacetate ${ }^{\mathrm{x}}$ & $6065-82-3$ & 852 & 1480 & $1487^{5}$ & 0.65 & 6.5 & $\mathrm{Nf}$ & - & $\mathrm{Nf}$ \\
\hline 130 & Ethyl 6-oxononanoate ${ }^{\mathrm{x}}$ & $4144-59-6$ & 841 & 1492 & $1488^{21}$ & 3.98 & 4.1 & $\mathrm{Nf}$ & - & $\mathrm{Nf}$ \\
\hline 131 & Methyl nonanoate $\mathrm{x}$ & $1731-84-6$ & 863 & 1494 & $1493^{28}$ & 9.87 & 9.6 & $\mathrm{Nf}$ & - & $\mathrm{Nf}$ \\
\hline 132 & Methyl 3-hydroxy-2-methylpropanoate ${ }^{z}$ & $72657-23-9$ & 837 & 1515 & $1507^{37}$ & 87.09 & 6.7 & $\mathrm{Nf}$ & - & Floral $^{71}$ \\
\hline 133 & Butyl 2-hydroxypropanoate (butyl lactate) ${ }^{z}$ & $138-22-7$ & 935 & 1522 & $1520^{21}$ & 230.78 & 5.6 & $1000^{49}$ & $<1$ & Creamy, milky, sweety ${ }^{49}$ \\
\hline 134 & Ethyl 2-hydroxyhexanoate ${ }^{z}$ & $6946-90-3$ & 966 & 1544 & $1542^{5}$ & 56.54 & 9.0 & $\mathrm{Nf}$ & - & Cashew, cherry ${ }^{34}$ \\
\hline 135 & Ethyl 2-hydroxy-4-methylpentanoate ${ }^{z}$ & $10348-47-7$ & 924 & 1554 & $1547^{34}$ & 91.86 & 3.9 & $51^{70}$ & 1.8 & Fruity, lemon ${ }^{34}$ \\
\hline 136 & Methyl 2-hydroxyhexanoate ${ }^{z}$ & 68756-64-9 & 981 & 1565 & $1574^{38}$ & 110.32 & 5.3 & $\mathrm{Nf}$ & - & $\mathrm{Nf}$ \\
\hline 137 & Diethyl propanedioate (diethyl malonate) ${ }^{\Delta}$ & $105-53-3$ & 861 & 1571 & $1580^{21}$ & 90.43 & 6.7 & $\mathrm{Nf}$ & - & $\mathrm{Nf}$ \\
\hline 138 & Ethyl nonanoate $^{\mathrm{x}}$ & $123-29-5$ & 841 & 1578 & $1581^{2}$ & 1603.71 & 5.2 & $1300^{57}$ & 1.2 & Floral, fruity ${ }^{8}$ \\
\hline 139 & Ethyl 3-hydroxypentanoate ${ }^{z}$ & $54074-85-0$ & 803 & 1589 & $1587^{38}$ & 16.98 & 6.3 & $\mathrm{Nf}$ & - & Fruity, pineapple-like ${ }^{38}$ \\
\hline 140 & Methyl decanoate ${ }^{\mathrm{x}}$ & $110-42-9$ & 804 & 1601 & $1599^{5}$ & 22.62 & 4.7 & $1.2^{57}$ & $<1$ & $\mathrm{Nf}$ \\
\hline 141 & Ethyl 4-oxopentanoate ${ }^{t}$ & $539-88-8$ & 944 & 1614 & $1607^{5}$ & 78.21 & 5.6 & $\mathrm{Nf}$ & - & $\mathrm{Nf}$ \\
\hline
\end{tabular}


Table 2 (continued)

\begin{tabular}{|c|c|c|c|c|c|c|c|c|c|c|}
\hline & Compound $^{\mathrm{a}}$ & CAS & $S^{b}$ & $\begin{array}{l}\text { LTPRI } \\
(\exp )^{\mathrm{c}}\end{array}$ & 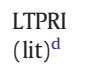 & $\begin{array}{l}\text { Conc. }^{\mathrm{e}} \\
\left(\mu \mathrm{g} \mathrm{L}^{-1}\right)\end{array}$ & $\begin{array}{l}\text { RSD } \\
(\%)^{f}\end{array}$ & $\begin{array}{l}\text { OTS } \\
\left(\mu \mathrm{g} \mathrm{L}^{-1}\right)^{\mathrm{h}}\end{array}$ & $O A V^{g}$ & Odor descriptor $^{\mathrm{h}}$ \\
\hline \multicolumn{11}{|l|}{ Esters } \\
\hline 142 & $\begin{array}{l}\text { 3-Methylbutyl 2-hydroxypropanoate } \\
\text { (isopentyl lactate/Isoamyl lactate) } \mathbf{( 1 0 )}\end{array}$ & 19329-89-6 & 936 & 1619 & $1615^{2}$ & 294.55 & 8.9 & $\mathrm{Nf}$ & - & $\mathrm{Nf}$ \\
\hline 143 & Butyl octanoate $(\mathbf{1 1})^{\mathrm{x}}$ & $589-75-3$ & 945 & 1624 & $1621^{28}$ & 145.63 & 2.3 & $700^{62, \mathrm{i}}$ & $<1$ & $\mathrm{Nf}$ \\
\hline 144 & $\begin{array}{l}\text { Methyl ethyl butanedioate } \\
\text { (methyl ethyl succinate) }(\mathbf{1 1})^{\Delta}\end{array}$ & $627-73-6$ & 978 & 1624 & $1631^{5}$ & 124.43 & 5.6 & $\mathrm{Nf}$ & - & $\mathrm{Nf}$ \\
\hline 145 & Ester $(\mathbf{1 2})^{\Delta}$ & - & 804 & 1641 & 1632 & 15.82 & 9.7 & - & - & - \\
\hline 146 & Ester $^{\Delta}$ & - & 807 & 1646 & 1635 & 40.32 & 9.4 & - & - & - \\
\hline 147 & Ethyl decanoate ${ }^{v}$ & $110-38-3$ & 925 & 1643 & $1651^{2}$ & 1950.32 & 8.6 & $500^{49}$ & 3.9 & Fruity, grape ${ }^{49}$ \\
\hline 148 & Ethyl benzoate ${ }^{\Upsilon}$ & $93-89-0$ & 958 & 1668 & $1664^{5}$ & 29.60 & 3.9 & $56^{55, i}$ & $<1$ & Fruity $^{41}$ \\
\hline 149 & Ester $^{\Upsilon}$ & & 817 & 1695 & $\mathrm{Nf}$ & 102.53 & 2.9 & - & - & - \\
\hline 150 & Ester $^{\Upsilon}$ & & 814 & 1744 & $\mathrm{Nf}$ & 32.31 & 5.4 & - & - & - \\
\hline 151 & 3-Methylbutyl octanoate (isoamyl octanoate) ${ }^{\mathrm{x}}$ & 2035-99-6 & 904 & 1668 & $1674^{2}$ & 54.96 & 3.7 & $152^{51}$ & $<1$ & Oily $^{3}$, sweet, light fruity, cheese, cream $^{51}$ \\
\hline 152 & Ethyl $(Z)$-4-decenoate $(\mathbf{1 4})^{\mathrm{x}}$ & $7367-84-2$ & 990 & 1690 & $1687^{16}$ & 45.65 & 3.8 & $\mathrm{Nf}$ & - & $\mathrm{Nf}$ \\
\hline 153 & Ester $^{\mathrm{x}}$ & - & 824 & 1702 & 1693 & 23.99 & 8.3 & - & - & - \\
\hline 154 & Methyl undecanoate ${ }^{\mathrm{x}}$ & $627-90-7$ & 908 & 1703 & $1694^{21}$ & 66.45 & 8.6 & $\mathrm{Nf}$ & - & $\mathrm{Nf}$ \\
\hline 155 & Diethyl butanedioate (diethyl succinate) $(\mathbf{1 4})^{\Delta}$ & $123-25-1$ & 952 & 1690 & $1701^{2}$ & 1680.31 & 6.8 & $1200^{49}$ & 1.4 & Fruity ${ }^{49}$ \\
\hline 156 & Ethyl 9-decenoate & $67233-91-4$ & 974 & 1708 & $1709^{40}$ & 191.29 & 2.8 & $100^{64}$ & 1.9 & Rose $^{3}$ \\
\hline 157 & Propyl decanoate ${ }^{\mathrm{x}}$ & $30673-60-0$ & 901 & 1711 & $1720^{5}$ & 29.73 & 5.4 & $\mathrm{Nf}$ & - & $\mathrm{Nf}$ \\
\hline 158 & Ethyl undecanoate $^{\mathrm{x}}$ & $627-90-7$ & 967 & 1739 & $1732^{22}$ & 102.43 & 7.2 & $\mathrm{Nf}$ & - & $\mathrm{Nf}$ \\
\hline 159 & Methyl phenylacetate ${ }^{\Upsilon}$ & $101-41-7$ & 964 & 1745 & $1749^{38}$ & 43.78 & 3.9 & $\mathrm{Nf}$ & - & $\mathrm{Nf}$ \\
\hline 160 & Methyl 2-hydroxybenzoate $^{\Upsilon}$ & $9041-28-5$ & 912 & 1755 & $1756^{41}$ & 77.40 & 6.4 & $\mathrm{Nf}$ & - & Minty, sweet ${ }^{41}$ \\
\hline 161 & Diethyl pentanedioate $\mathrm{x}^{\mathrm{x}}$ & $818-38-2$ & 945 & 1770 & $1768^{22}$ & 76.37 & 9.5 & $\mathrm{Nf}$ & - & $\mathrm{Nf}$ \\
\hline 162 & Ester $^{\mathrm{x}}$ & - & 819 & 1789 & $\mathrm{Nf}$ & 50.64 & 6.0 & - & - & - \\
\hline 163 & Methyl dodecanoate ${ }^{\mathrm{x}}$ & $111-82-0$ & 904 & 1800 & $1793^{22}$ & 22.19 & 3.8 & $\mathrm{Nf}$ & - & $\mathrm{Nf}$ \\
\hline 164 & Ethyl 2-phenylacetate (20) ${ }^{\Upsilon}$ & $103-45-7$ & 985 & 1796 & $1805^{22}$ & 3420.04 & 7.5 & $1800^{49}$ & 1.9 & Flowery ${ }^{49}$ \\
\hline 165 & Isopropyl dodecanoate $\mathrm{x}^{\mathrm{x}}$ & $10233-13-3$ & 946 & 1812 & $1821^{40}$ & 34.60 & 4.9 & $\mathrm{Nf}$ & - & $\mathrm{Nf}$ \\
\hline 166 & Ethyl 3,3-diethoxypropionate ${ }^{\mathrm{x}}$ & $10601-80-6$ & 917 & 1833 & $1829^{5}$ & 84.54 & 2.6 & $\mathrm{Nf}$ & - & $\mathrm{Nf}$ \\
\hline 167 & Ethyl dodecanoate (ethyl laurate) ${ }^{\mathrm{x}}$ & $106-33-2$ & 928 & 1839 & $1835^{22}$ & 5604.11 & 3.1 & $3500^{53}$ & 1.6 & Sweet, floral, fruity cream ${ }^{57}$ \\
\hline 168 & Ester $^{x}$ & & 814 & 1845 & $\mathrm{Nf}$ & 10.93 & 8.6 & $\mathrm{Nf}$ & - & $\mathrm{Nf}$ \\
\hline 169 & Isopentyl decanoate $\mathrm{e}^{\mathrm{x}}$ & $2306-91-4$ & 938 & 1868 & $1871^{2}$ & 33.65 & 8.9 & $\mathrm{Nf}$ & - & $\mathrm{Nf}$ \\
\hline 170 & Methyl 3-hydroxybutanoate ${ }^{z}$ & $20257-95-8$ & 937 & 1882 & $1880^{19}$ & 5400.88 & 3.6 & $\mathrm{Nf}$ & - & $\mathrm{Nf}$ \\
\hline 171 & Ester $^{\Delta}$ & - & 821 & 1879 & $\mathrm{Nf}$ & 43.60 & 5.2 & - & - & - \\
\hline 172 & Ester $^{\Delta}$ & - & 809 & 1890 & $\mathrm{Nf}$ & 100.15 & 6.9 & - & - & - \\
\hline 173 & Ethyl tridecanoate $^{\mathrm{x}}$ & 28267-29-0 & 928 & 1951 & $1943^{21}$ & 67.09 & 1.9 & $\mathrm{Nf}$ & - & $\mathrm{Nf}$ \\
\hline 174 & Methyl tetradecanoate ${ }^{\mathrm{x}}$ & $124-10-7$ & 964 & 2021 & $2034^{30}$ & 49.09 & 6.5 & $\mathrm{Nf}$ & - & Waxy $^{30}$ \\
\hline 175 & Diethyl 2-hydroxybutanedioate (diethyl malate) ${ }^{\Delta}$ & $7554-12-3$ & 948 & 2048 & $2048^{5}$ & 1500.92 & 6.7 & $760,000^{32}$ & $<1$ & Over-ripe, peach, cut grass ${ }^{32}$ \\
\hline 176 & Ethyl tetradecanoate (isopropyl myristate) ${ }^{\mathrm{x}}$ & $124-06-1$ & 987 & 2059 & $2054^{5}$ & 43.09 & 2.3 & $800^{57}$ & $<1$ & $\mathrm{Nf}$ \\
\hline 177 & Hexyl benzoate $^{\Upsilon}$ & $6789-88-4$ & 925 & 2057 & $2056^{6}$ & 90.31 & 8.9 & $\mathrm{Nf}$ & - & $\mathrm{Nf}$ \\
\hline 178 & 2-Phenylethyl hexanoate ${ }^{\Upsilon}$ & $6290-37-5$ & 928 & 2157 & $2160^{8}$ & 15.66 & 4.4 & $\mathrm{Nf}$ & - & $\mathrm{Nf}$ \\
\hline 179 & Ethyl pentadecanoate $(\mathbf{1 6})^{\mathrm{x}}$ & $41114-00-5$ & 967 & 2149 & $2151^{5}$ & 134.33 & 6.7 & $\mathrm{Nf}$ & - & $\mathrm{Nf}$ \\
\hline 180 & Dimethyl decanedioate $\mathrm{e}^{\mathrm{x}}$ & $106-79-6$ & 899 & 2209 & $2220^{5}$ & 120.18 & 6.6 & $\mathrm{Nf}$ & - & $\mathrm{Nf}$ \\
\hline 181 & Ethyl hexadecanoate $\mathrm{x}^{\mathrm{x}}$ & $628-97-7$ & 904 & 2250 & $2261^{5}$ & 150.22 & 3.4 & $1500^{51}$ & $<1$ & Fatty, rancid, fruity, sweet ${ }^{51}$ \\
\hline 182 & Ethyl 9-hexadecenoate ${ }^{\mathrm{x}}$ & $54546-22-4$ & 912 & 2289 & $2283^{5}$ & 9.08 & 9.0 & $\mathrm{Nf}$ & - & $\mathrm{Nf}$ \\
\hline 183 & 2-Phenethyl octanoate ${ }^{\Upsilon}$ & $5457-70-5$ & 927 & 2378 & $2376^{21}$ & 30.97 & 5.6 & $\mathrm{Nf}$ & - & $\mathrm{Nf}$ \\
\hline 184 & Ethyl octadecanoate ${ }^{\mathrm{x}}$ & $111-61-5$ & 988 & $2445^{j}$ & $2458^{21}$ & 192.10 & 7.8 & $\mathrm{Nf}$ & - & $\mathrm{Nf}$ \\
\hline 185 & $\begin{array}{l}\text { Ethyl 4-hydroxy-3-methoxybenzoate } \\
\text { (ethyl vanillate) }^{\Upsilon}\end{array}$ & $617-05-0$ & 901 & $2651^{\mathrm{j}}$ & $2665^{26}$ & 5100.09 & 6.4 & $3000^{65}$ & 1.7 & Sweet, honey, vanillin ${ }^{32}$ \\
\hline \multicolumn{11}{|c|}{ Ketones } \\
\hline 186 & 2-Propanone (acetone) ${ }^{\mathrm{r}}$ & $67-64-1$ & 907 & $815^{\mathrm{j}}$ & $816^{11}$ & 58.32 & 9.4 & $\mathrm{Nf}$ & - & $\mathrm{Nf}$ \\
\hline 187 & 4-Methyl-2-hexanone ${ }^{\mathrm{r}}$ & $110-12-3$ & 889 & $832^{\mathrm{j}}$ & $825^{42}$ & 34.01 & 5.7 & $\mathrm{Nf}$ & - & $\mathrm{Nf}$ \\
\hline 188 & 2-Butanone $e^{r}$ & $78-93-3$ & 945 & 908 & $903^{42}$ & 22.56 & 5.8 & $80,000^{53, i}$ & $<1$ & Acetone-like 53 \\
\hline 189 & 2,3-Butanedione ${ }^{\mathrm{r}}$ & $431-03-8$ & 908 & 971 & $975^{42}$ & 187.19 & 7.3 & $100^{50}$ & 8.7 & Buttery $^{3}$ \\
\hline 190 & 1-Penten-3-one $\mathrm{r}^{\mathrm{r}}$ & $1629-58-9$ & 975 & 1000 & $1008^{11}$ & 33.82 & 5.7 & $\mathrm{Nf}$ & - & Plastic, pungent ${ }^{47}$ \\
\hline 191 & Cyclopentanone $^{\mathrm{r}}$ & $120-92-3$ & 963 & 1146 & $1144^{1}$ & 34.80 & 8.9 & $\mathrm{Nf}$ & - & $\mathrm{Nf}$ \\
\hline 192 & 5-Methyl-3-heptanone (2) ${ }^{\mathrm{s}}$ & $541-85-5$ & 954 & 1260 & $1264^{33}$ & 10.67 & 7.8 & $\mathrm{Nf}$ & - & $\mathrm{Nf}$ \\
\hline 193 & 3-Octanone $\mathrm{s}^{\mathrm{s}}$ & $106-68-3$ & 907 & 1271 & $1266^{2}$ & 10.34 & 2.9 & $21.4^{55, \mathrm{i}}$ & $<1$ & Herbal $^{55}$ \\
\hline 194 & 3-Hydroxy-2-butanone (acetoin) $)^{\mathrm{u}}$ & $513-86-0$ & 968 & 1279 & $1287^{5}$ & 180723.42 & 2.6 & $150,000^{49}$ & 1.2 & Buttery, cream $^{49}$ \\
\hline 195 & 1-Hydroxy-2-propanone ${ }^{\mathrm{u}}$ & $116-09-6$ & 966 & 1315 & $1323^{43}$ & 23.05 & 7.9 & $10^{60}$ & $<1$ & $\mathrm{Nf}$ \\
\hline 196 & Cyclohexanone $\mathrm{s}^{\mathrm{s}}$ & $108-94-1$ & 915 & 1276 & $1285^{6}$ & 9.83 & 4.6 & $\mathrm{Nf}$ & - & $\mathrm{Nf}$ \\
\hline 197 & 1-Octen-3-one $e^{\mathrm{s}}$ & $4312-99-6$ & 937 & 1300 & $1299^{28}$ & 4.88 & 6.6 & $15^{65}$ & $<1$ & Mushroom $^{41}$ \\
\hline 198 & 6-Methyl-5-hepten-2-one $\mathrm{s}^{\mathrm{s}}$ & $110-93-0$ & 897 & 1349 & $1338^{42}$ & 30.74 & 0.9 & $68^{55, i}$ & $<1$ & Sweet, fruity ${ }^{55}$ \\
\hline 199 & 4-Hydroxy-4-methyl-2-pentanone (3) ${ }^{\mathrm{q}}$ & $123-42-2$ & 981 & 1351 & $1339^{40}$ & 64.03 & 0.5 & $\mathrm{Nf}$ & - & $\mathrm{Nf}$ \\
\hline 200 & 2-Nonanone $\mathrm{q}^{\mathrm{q}}$ & $821-55-6$ & 884 & 1391 & $1394^{28}$ & 61.50 & 7.5 & $41^{53}$ & 1.5 & Fruity ${ }^{53}$ \\
\hline 201 & 3-Decanone $e^{\mathrm{q}}$ & $928-80-3$ & 922 & 1482 & $1491^{14}$ & 20.22 & 4.3 & $\mathrm{Nf}$ & - & $\mathrm{Nf}$ \\
\hline 202 & 3-Methyl-2-cyclohexenone ${ }^{\Upsilon}$ & $1193-18-6$ & 910 & 1565 & $1579^{6}$ & 421.32 & 3.3 & $\mathrm{Nf}$ & - & $\mathrm{Nf}$ \\
\hline 203 & 2,3-Dimethyl-2-cyclopenten-1-one ${ }^{\Upsilon}$ & $1121-05-7$ & 928 & 1571 & $1582^{43}$ & 17.44 & 8.4 & $\mathrm{Nf}$ & - & $\mathrm{Nf}$ \\
\hline 204 & 2-Hydroxy-2-cyclopenten-1-one ${ }^{\Upsilon}$ & $21835-01-8$ & 934 & 1780 & $1784^{39}$ & 142.32 & 4.9 & $\mathrm{Nf}$ & - & $\mathrm{Nf}$ \\
\hline 205 & 2-Dodecanone & $6175-49-1$ & 991 & 1803 & $1809^{44}$ & 3.21 & 0.7 & $\mathrm{Nf}$ & - & $\mathrm{Nf}$ \\
\hline \multicolumn{11}{|c|}{ C13-norisoprenoids } \\
\hline 206 & $\beta$-Damascenone (17) ${ }^{+}$ & $23696-85-7$ & 909 & 1839 & $1842^{33}$ & 1.08 & 3.7 & $0.05^{50}$ & 20 & Honey, sweet ${ }^{3}$ \\
\hline 207 & Geranylcetone & $3796-70-1$ & 926 & 1850 & $1856^{5}$ & 9.72 & 5.8 & $\mathrm{Nf}$ & - & Floral $^{41}$ \\
\hline 208 & Methyl dihydrojasmonate & $24851-98-7$ & 938 & 2280 & $2276^{23}$ & 7.23 & 1.3 & $\mathrm{Nf}$ & - & $\mathrm{Nf}$ \\
\hline
\end{tabular}


Table 2 (continued)

\begin{tabular}{|c|c|c|c|c|c|c|c|c|c|c|}
\hline & Compound $^{\mathrm{a}}$ & CAS & $\mathrm{S}^{\mathrm{b}}$ & $\begin{array}{l}\text { LTPRI } \\
(\exp )^{\mathrm{c}}\end{array}$ & $\begin{array}{l}\text { LTPRI } \\
\text { (lit) }^{d}\end{array}$ & $\begin{array}{l}\text { Conc. }^{\mathrm{e}} \\
\left(\mu \mathrm{g} \mathrm{L}^{-1}\right)\end{array}$ & $\begin{array}{l}\text { RSD } \\
(\%)^{\mathrm{f}}\end{array}$ & $\begin{array}{l}\text { OTS } \\
\left(\mu \mathrm{g} \mathrm{L}^{-1}\right)^{\mathrm{h}}\end{array}$ & $\mathrm{OAV}^{\mathrm{g}}$ & Odor descriptor ${ }^{\mathrm{h}}$ \\
\hline \multicolumn{11}{|c|}{ Terpenes } \\
\hline 209 & 3-Carene & $13466-78-9$ & 901 & 1134 & $1146^{1}$ & 70.40 & 2.3 & $44^{69, \mathrm{i}}$ & 1.6 & Mango leaf-like, sweet, green ${ }^{69}$ \\
\hline 210 & $\rho$-Cymene & $99-87-6$ & 893 & 1250 & $1262^{38}$ & 27.36 & 5.4 & $11.40^{62, \mathrm{i}}$ & 2.4 & Fruity, sweet ${ }^{30}$ \\
\hline 211 & 3,3,5-Trimethyl-2-cyclohexen-1-one (isoforone) & $78-59-1$ & 981 & 1393 & $1381^{21}$ & 8.65 & 7.8 & $\mathrm{Nf}$ & - & $\mathrm{Nf}$ \\
\hline 212 & (Z)-Linaloloxide & 5989-33-3 & 888 & 1429 & $1438^{38}$ & 19.29 & 8.3 & $500^{51}$ & $<1$ & Rose, wood $^{51}$ \\
\hline 213 & Hotrienol & $29957-43-5$ & 934 & 1460 & $1449^{13}$ & 8.67 & 2.3 & $\mathrm{Nf}$ & - & $\mathrm{Nf}$ \\
\hline 214 & Linalool ${ }^{\bullet}$ & $78-70-6$ & 907 & 1539 & $1540^{22}$ & 28.50 & 3.7 & $15^{49}$ & 1.9 & Citrus, floral, sweet, grape-like ${ }^{49}$ \\
\hline 215 & $\beta$-Elemene (9) & $515-13-9$ & 952 & 1600 & $1595^{30}$ & 5.44 & 5.3 & $\mathrm{Nf}$ & - & $\mathrm{Nf}$ \\
\hline 216 & 4-Terpineol & $562-74-3$ & 887 & 1609 & $1602^{5}$ & 40.82 & 6.3 & $250^{68}$ & $<1$ & Sweet, herbaceous ${ }^{41}$ \\
\hline 217 & $\alpha$-Terpineol & $98-55-5$ & 899 & 1713 & $1706^{33}$ & 1100.67 & 5.4 & $1000^{49}$ & 1.1 & Floral, sweet ${ }^{49}$ \\
\hline 218 & Dihydrocarveol` & $38049-26-2$ & 936 & 1715 & $1720^{45}$ & 4.21 & 3.3 & $100^{50}$ & $<1$ & $\mathrm{Nf}$ \\
\hline 219 & $\alpha$-Citronellol ${ }^{\star}$ & $106-22-9$ & 907 & 1774 & $1771^{30}$ & 123.98 & 6.3 & $100^{50}$ & 1.2 & Sweet, citrus-like $\mathrm{e}^{30}$ \\
\hline 220 & Nerol ${ }^{\star}$ & $106-25-2$ & 922 & 1785 & $1797^{5}$ & 34.21 & 4.2 & $400^{67}$ & $<1$ & Rose, lime ${ }^{67}$ \\
\hline 221 & $\beta$-Ionone & $106-28-5$ & 883 & 1920 & $1924^{41}$ & 12.93 & 5.3 & $5^{49}$ & $<1$ & Balsamic, rose, violet ${ }^{49}$ \\
\hline 222 & (E)-Nerolidol ${ }^{\bullet}$ & $40716-66-3$ & 884 & 2062 & $2058^{2}$ & 1630.22 & 8.7 & $1000^{49}$ & 1.6 & Rose, apple, green, citrus ${ }^{49}$ \\
\hline 223 & (E)-Farnesol & $4602-84-0$ & 931 & 2335 & $2345^{26}$ & 2881.10 & 2.6 & $2400^{49}$ & 1.2 & Floral $^{49}$ \\
\hline 224 & (Z)- $\alpha$-Santalol ${ }^{\bullet}$ & $115-71-9$ & 928 & 2348 & $2350^{47}$ & 33.90 & 7.4 & $\mathrm{Nf}$ & - & Incense, woody ${ }^{47}$ \\
\hline \multicolumn{11}{|c|}{ Phenols } \\
\hline 225 & 2-Methoxy phenol (guaiacol) ${ }^{+}$ & $90-05-1$ & 907 & 1861 & $1860^{10}$ & 3.87 & 5.2 & $10^{50}$ & $<1$ & Smoke, sweet, medicine ${ }^{67}$ \\
\hline 226 & 4-Methylguaiacol ${ }^{+}$ & $108-95-2$ & 894 & 2002 & $2007^{8}$ & 8.90 & 4.5 & $\mathrm{Nf}$ & - & $\mathrm{Nf}$ \\
\hline 227 & 4-Ethylguaiacol ${ }^{+}$ & $2785-89-9$ & 881 & 2039 & $2033^{5}$ & 3.67 & 2.1 & $33^{51}$ & $<1$ & Medicine, wood, clove, smoky ${ }^{51}$ \\
\hline 228 & Eugenol $^{+}$ & $97-53-0$ & 963 & 2155 & $2157^{5}$ & 1.29 & 1.8 & $5^{50}$ & $<1$ & Spices, clove, honey ${ }^{32}$ \\
\hline 229 & 4-Ethylphenol ${ }^{+}$ & $123-07-9$ & 914 & 2154 & $2167^{38}$ & 2.04 & 0.9 & $610^{52}$ & $<1$ & Phenolic ${ }^{41}$ \\
\hline 230 & 4-Vinylguaiacol ${ }^{+}$ & $7786-61-0$ & 915 & 2195 & $2190^{19}$ & 5.80 & 4.5 & $40^{50}$ & $<1$ & Spices, curry ${ }^{32}$ \\
\hline 231 & 5-Isopropyl-3-methylphenol ${ }^{+}$ & $3228-03-3$ & 847 & 2282 & $2287^{45}$ & 2.89 & 0.9 & $\mathrm{Nf}$ & - & $\mathrm{Nf}$ \\
\hline \multicolumn{11}{|c|}{ Pyrroles } \\
\hline 232 & Ethyl-pyrrole & $617-92-5$ & 991 & 1198 & $1194^{43}$ & 8.93 & 9.3 & $\mathrm{Nf}$ & - & $\mathrm{Nf}$ \\
\hline 233 & Pyrrole-2-carboxaldehyde & $1003-29-8$ & 974 & 1996 & $1990^{39}$ & 16.78 & 4.1 & $\mathrm{Nf}$ & - & $\mathrm{Nf}$ \\
\hline 234 & 2-Pyrrolidinone & $616-45-5$ & 892 & 2037 & $2041^{46}$ & 19.22 & 4.7 & $\mathrm{Nf}$ & - & $\mathrm{Nf}$ \\
\hline \multicolumn{11}{|c|}{ Sulfur compounds } \\
\hline 235 & Ethyl (methylthio)acetate (8) ${ }^{\mathrm{q}}$ & $4455-13-4$ & 814 & 1450 & $1438^{38}$ & 20.76 & 7.2 & $\mathrm{Nf}$ & - & Cucumber-like ${ }^{66}$ \\
\hline 236 & Ethyl 3-(methylthio)propanoate ${ }^{q}$ & $13532-18-8$ & 917 & 1566 & $1558^{38}$ & 18.91 & 5.6 & $7^{63}$ & 2.7 & Pineapple-like $e^{63}$ \\
\hline 237 & 3-(Methylthio)-1-propanol (15) $)^{\Phi}$ & $505-10-2$ & 965 & 1729 & $1738^{2}$ & 28.35 & 4.4 & $500^{50}$ & $<1$ & Cooked vegetable $\mathrm{e}^{32}$ \\
\hline 238 & 2-Thiophenecarboxaldehyde $(\mathbf{1 5})^{\mathrm{q}}$ & $98-03-3$ & 985 & 1729 & $1734^{43}$ & 30.32 & 2.1 & $\mathrm{Nf}$ & - & $\mathrm{Nf}$ \\
\hline \multicolumn{11}{|c|}{ Furans } \\
\hline 239 & 2-Methyl-furan & $534-22-5$ & 892 & 861 & $858^{43}$ & 4.32 & 1.9 & $\mathrm{Nf}$ & - & $\mathrm{Nf}$ \\
\hline 240 & 2-Pentyl-furan (1) & 3777-69-3 & 935 & 1246 & $1240^{10}$ & 5.87 & 0.9 & $6^{60, i}$ & $<1$ & Sweet $^{12}$ \\
\hline 241 & 2-(Diethoxymethyl)furan & $13529-27-6$ & 987 & 1438 & $1442^{8}$ & 75.01 & 4.3 & $\mathrm{Nf}$ & - & Roasted $^{8}$ \\
\hline 242 & Ethyl 2-furoate ${ }^{\Upsilon}$ & $614-99-3$ & 965 & 1617 & $1618^{5}$ & 235.43 & 7.4 & $16,000^{65}$ & $<1$ & Balsamic $^{8}$ \\
\hline 243 & 2-Furanmethanol ${ }^{\bullet}$ & $98-00-0$ & 981 & 1640 & $1647^{8}$ & 32.43 & 2.8 & $\mathrm{Nf}$ & - & Burnt sugar ${ }^{8}$ \\
\hline
\end{tabular}

CAS: Chemical Abstract Service.

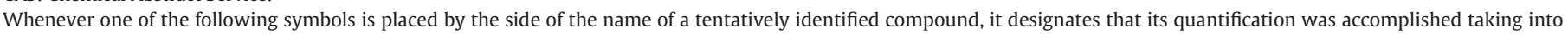

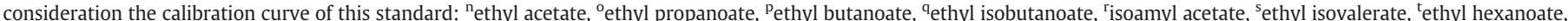

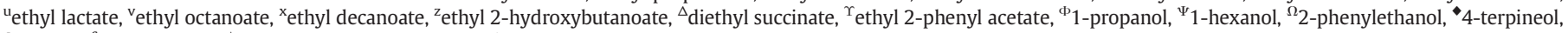

+eugenol, ${ }^{\S}$ hexanoic acid, ${ }^{\star}$ octanoic acid, ${ }^{\infty}$ decanoic acid, ${ }^{*}$ dodecanoic acid.

a Co-elutions were numbered from 1 to 20 and these numbers are written between parentheses after the compound's name. Whenever compounds are followed by the same number, they co-elute.

b Similarity.

c LTPRI exp: Experimental linear temperature programmed retention index (LTPRI) calculated using $n$-alkanes (C9-C24) in polar $\times($ DB-Wax, $100 \%$ polyethyleneglycol) $\times$ medium polar (DB-17 ms, (50\%-phenyl)-methylpolysiloxane) column set.

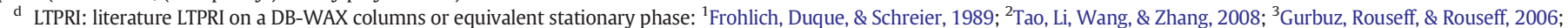

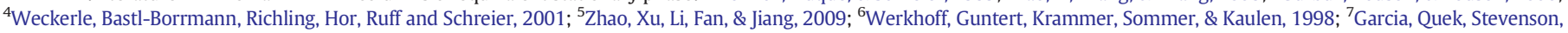

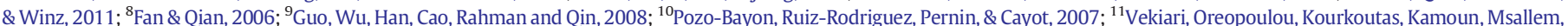

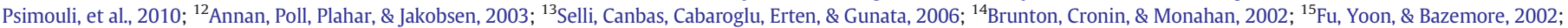

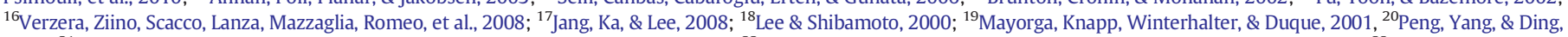

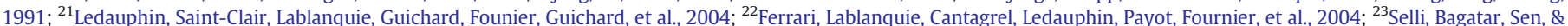

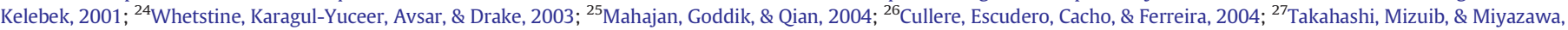

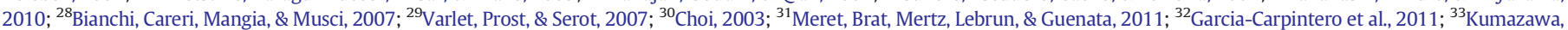

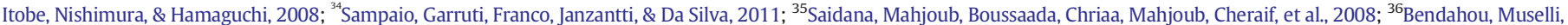

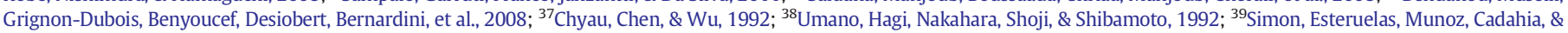

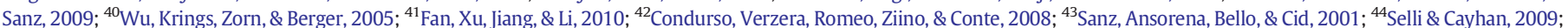

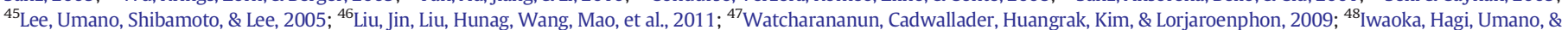
Shibamoto, 1994.

e Concentration.

f Relative standard deviation.

g Odor activity value.

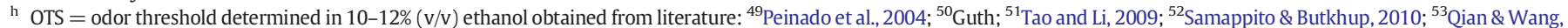

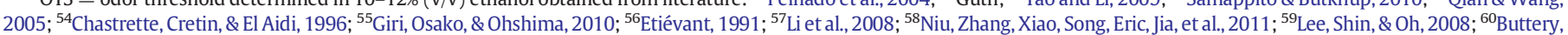

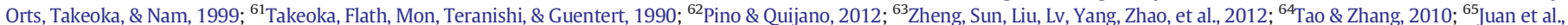
2012; ${ }^{66}$ Kourkoutas et al., 2006; ${ }^{67}$ Vilanova et al., 2010; ${ }^{68}$ Ferreira et al., 2000; ${ }^{69}$ Boonbumrung et al., 2001; ${ }^{70}$ Lytra, Tempere, Revel, \& Barbe, $2012 ;{ }^{71}$ Morales \& Duque, 2002.

i Odor threshold obtained in water.

j Extrapolated LTPRI for compounds with LTPRI $<900$ and $>2400$.

${ }^{\mathrm{k}}$ Not found: LTPRI, odor threshold or odor descriptor are not available in the literature. 
1996). Calibration graphs with at least six concentration levels for each standard compound were constructed by least square linear regression. The relative area of each compound (area compound/area internal standard) was plotted against the respective compound concentration. For each standard compound a calibration curve equation, determination coefficient $\left(r^{2}\right)$, and linear range were calculated.

Determination of the LOD and LOQ were based on the standard deviation the intersection of the analytical curve (s) and on the slope of the curve (S). The LOD was expressed as $3.3(\mathrm{~s} / \mathrm{S})$ and LOQ as 10 times $(\mathrm{s} / \mathrm{S})$.

The precision of the method was evaluated in terms of repeatability and intermediate precision expressed as relative standard deviation (RSD). Precision was evaluated as repeatability by carrying out six independent assays performed under the same analytical conditions in a short period of time on the same day, whereas intermediate precision was determined on two different days. Precision and accuracy assays were determined for two levels of concentration, including the lower and the higher concentrations of the calibration curves. Accuracy has been evaluated by addition of standard volatile compounds in a model wine solution and was determined after five extractions and analysis procedures for each level of concentration, for each one of the 22 standard compounds. It was calculated as the percentage deviation between the calculated value and the nominal value obtained in the calibration curves.

Quantitative data for the 22 positively identified compounds (Table 1 ) were obtained by interpolation of the calibration graphs built with pure reference compounds. The concentration of the tentatively identified volatile compounds, for which there was no pure reference, was obtained using calibration graphs of the available standard compounds. In this case, calibration graphs were chosen according to the chemical structure of the tentatively identified volatile compound to be quantified, trying to choose the standard compound that would be most similar to the tentatively identified one. The linear regression equations and other parameters concerning the calibration curves of the standard compounds that were used in the quantification of each volatile compound are indicated in Table 1.

\subsection{Odor activity value (OAV) and relative odor contribution (ROC)}

OAV was calculated by dividing the mean concentration $(n=5)$ of a compound by its odor threshold value, published in the scientific literature. Odor thresholds obtained in hydroalcoholic solution (matrix similar to wine with $10-12 \%$ ethanol) were used whenever available in the scientific literature.

\section{Results and discussion}

\subsection{Identification of volatile compounds}

In the headspace of Chardonnay wines, 243 compounds were tentatively identified using GC $\times$ GC/TOFMS, and they are presented in Table 2, according to their chemical classes and in order of increasing LTPRI. Among them, 22 compounds were positively identified using standard compounds. Similarity values between the mass spectra of sample compounds and NIST library, as well as calculated LTPRI and literature LTPRI for each compound are also shown in Table 2. All these data have contributed to the tentative identification of volatile compounds. LTPRI from scientific literature have been found in 1D-GC papers and this approach has been already discussed in a previous work of this research group (Welke, Manfroi, et al., 2012) Differences between calculated LTPRI and literature LTPRI were accepted for the process of tentative identification when they were less than 14 units. Only $13 \%$ of the tentatively identified compounds showed LTPRI values greater than 10 units. Some of them were acids, including 4-methyl-2oxovaleric acid, isobutyric acid, hexanoic acid and heptanoic acid, which have presented the highest differences between experimental and literature LTPRI. It is well known that polar column LTPRI are more prone to variations (von Muhlen et al., 2008), and in the case of this work, some variability could be expected, as two polar columns were coupled.

Some studies about volatile composition of Chardonnay wines using 1D-GC have been published and the number of compounds tentatively identified was approximately 50 (Jaffré et al., 2011; Jiang \& Zhang, 2010; Li et al., 2008). This number suggests that former GC/MS methods were able to identify only part of the volatile compounds that may be identified when GC $\times$ GC/TOFMS is employed in Chardonnay wine headspace analysis.

Among all the chemical groups found in the volatile content of Chardonnay wines of Serra Gaúcha, esters were present in higher number (85), followed by alcohols (52), acids (26), aldehydes (22), ketones (20), terpenes (16), phenols (7), furans (5), sulfur compounds (4), pyrroles (3) and C13-norisoprenoids (3). Predominant presence of alcohols, esters and acids in Chardonnay wine has already been observed in previous studies, although in this case, the number of alcohols was higher than that of esters (Jiang \& Zhang, 2010; Li et al., 2008).

A color plot obtained of a Chardonnay wine analyzed by HS-SPME-GC $\times$ $\mathrm{GC} / \mathrm{TOFMS}$ is shown in Fig. 1A. Some co-elutions that might have happened with the use of one-dimensional GC can be viewed in this color plot. Forty-two compounds co-eluted in ${ }^{1} \mathrm{D}$ and 8 of them also coeluted in ${ }^{2} \mathrm{D}$. In Table 2, compounds that co-elute with other components are numbered and this number is informed after the compound name, between parentheses. Co-elutions are numbered from (1) until (20).

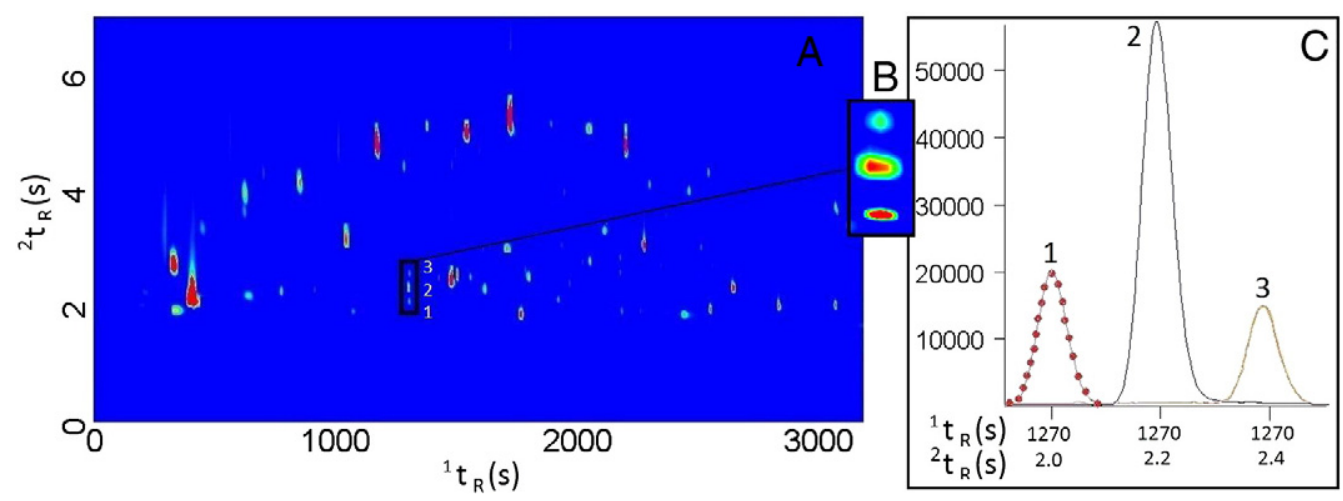

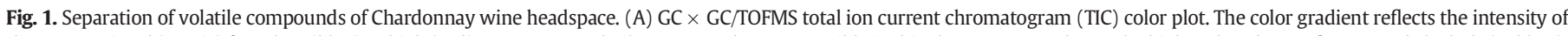

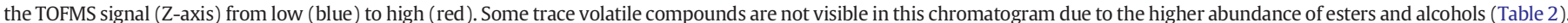

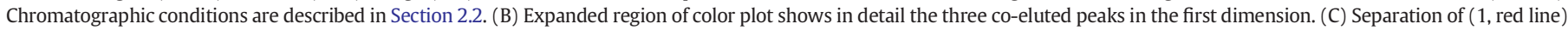

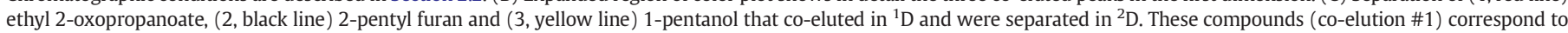
numbers 112, 240 and 7 in Table 2. 
An interesting example of co-elution of compounds of the headspace of Chardonnay wines in the first dimension $\left({ }^{1} t_{R}=1270 \mathrm{~s}\right)$ and also of their separation in the second dimension is given by co-elution (1), which includes 1-pentanol (\# 7 in Table $2,{ }^{2} t_{R}=2.4 \mathrm{~s}$, LTPRI experimental 1245, LTPRI of literature 1233 (Weckerle et al., 2001), 2-pentyl-furan (\# 240 of Table $2,{ }^{2} t_{R}=2.2 \mathrm{~s}$, LTPRI experimental 1246, LTPRI of literature 1240 (Pozo-Bayon et al., 2007) and ethyl 2-oxopropanoate (\# 112 in Table $2,{ }^{2} t_{R}=2.0 \mathrm{~s}$, LTPRI experimental 1247, LTPRI of literature 1242 (Selli et al., 2006). These three co-eluting compounds are highlighted in the color plot of Fig. $1 \mathrm{~A}$ and their separation in the second dimension is shown in detail in Fig. 1B. Regarding their contribution to wine aroma, 1-pentanol can contribute negatively to wine aroma because its odor perception is described as synthetic and balsamic (Garcia-Carpintero et al., 2011). Another co-eluting compound, 2-pentylfuran, may have a positive contribution to aroma, as its odor description is known as sweet (Annan et al., 2003). No information was found in the scientific literature about the contribution of ethyl 2-oxopropanoate to wine aroma. This example highlights the importance of resolution enhancement provided by GC $\times$ GC, whenever tentative identification of aroma active compounds that may co-elute in the first dimension is necessary.

\subsection{Method validation}

The performance of the method in terms of linearity, limits of detection (LOD) and quantification (LOQ), precision (repeatability and intermediate precision) and accuracy are shown in Tables 1 and 3.

The choice of the concentration ranges for each volatile compound was based on previously published works (Guth, 1997; Juan et al., 2012; Li et al., 2008; Vilanova et al., 2010). Preliminary analyses were performed to verify if the concentration of volatile compounds found in wines from Serra Gaucha was within the range of concentrations used in the calibration curves. In some cases, such as ethyl acetate, ethyl hexanoate and ethyl octanoate, the range of concentration had to be adjusted. In the case of ethyl acetate, the concentration found in the Serra Gaucha samples was lower than that previously reported and for ethyl hexanoate and ethyl octanoate, the levels were higher than in other works (Guth, 1997; Juan et al., 2012; Li et al., 2008; Vilanova et al., 2010).

The calibration curves were found to have good linearity in the range of studied concentrations. The determination coefficient $\left(R^{2}\right)$ values were in the range of 0.998 to 0.972 for the evaluated standard compounds (Table 1 ), with the exception of organic acids that presented

Table 3

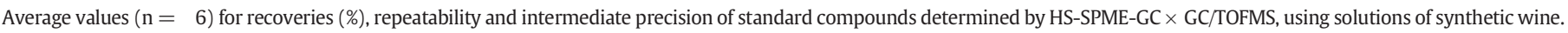

\begin{tabular}{|c|c|c|c|c|}
\hline Compound & Concentration $\left(\mu \mathrm{g} \mathrm{L}^{-1}\right)$ & Recovery (\%) & Repeatability RSD (\%) ${ }^{\mathrm{a}}$ & Intermediate precision RSD $(\%)^{\mathrm{b}}$ \\
\hline \multirow[t]{2}{*}{ Ethyl acetate } & 9610 & 99.3 & 2.6 & 4.5 \\
\hline & 20,880 & 98.3 & 9.3 & 9.9 \\
\hline \multirow[t]{2}{*}{ Ethyl propanoate } & 1200 & 99.8 & 1.6 & 3.4 \\
\hline & 20,000 & 99.8 & 9.6 & 9.8 \\
\hline \multirow[t]{2}{*}{ Ethyl butanoate } & 2500 & 98.6 & 5.5 & 6.4 \\
\hline & 25,000 & 95.7 & 3.4 & 4.5 \\
\hline \multirow[t]{2}{*}{ Ethyl 2-methyl propanoate (ethyl isobutanoate) } & 12.0 & 97.7 & 5.4 & 3.3 \\
\hline & 168 & 98.1 & 8.7 & 6.7 \\
\hline \multirow[t]{2}{*}{ 3-Methyl butyl acetate (isoamyl acetate } & 18.0 & 96.4 & 2.9 & 4.5 \\
\hline & 490.0 & 95.5 & 4.4 & 5.1 \\
\hline \multirow[t]{2}{*}{ Ethyl 2-methylbutanoate (ethyl isovalerate) } & 3.5 & 95.5 & 8.5 & 5.7 \\
\hline & 56 & 96.2 & 2.5 & 3.2 \\
\hline \multirow[t]{2}{*}{ Ethyl hexanoate } & 58 & 97.3 & 8.9 & 9.1 \\
\hline & 660 & 98.7 & 5.7 & 6.4 \\
\hline \multirow[t]{2}{*}{ Ethyl 2-hydroxy propanoate (ethyl lactate) } & 59,000 & 99.2 & 5.7 & 5.5 \\
\hline & 688,800 & 96.8 & 4.8 & 5.3 \\
\hline \multirow[t]{2}{*}{ Ethyl octanoate } & 52,000 & 96.1 & 7.8 & 3.2 \\
\hline & 260,000 & 102.6 & 4.0 & 6.5 \\
\hline \multirow[t]{2}{*}{ Ethyl decanoate } & 180 & 95.7 & 1.2 & 2.8 \\
\hline & 7840 & 97.5 & 7.7 & 7.8 \\
\hline \multirow[t]{2}{*}{ Ethyl 2-hydroxy-butanoate } & 4500 & 96.3 & 5.7 & 6.5 \\
\hline & 72,700 & 95.7 & 3.3 & 3.7 \\
\hline \multirow[t]{2}{*}{ Diethyl succinate } & 24.88 & 98.6 & 6.7 & 7.1 \\
\hline & 2300 & 93.7 & 2.4 & 3.4 \\
\hline \multirow[t]{2}{*}{ Ethyl 2-phenyl acetate } & 14.3 & 96.3 & 1.6 & 2.3 \\
\hline & 460 & 95.4 & 7.9 & 7.8 \\
\hline \multirow[t]{2}{*}{ 1-Propanol } & 15 & 99.7 & 1.3 & 3.2 \\
\hline & 192 & 97.7 & 3.8 & 3.9 \\
\hline \multirow[t]{2}{*}{ 1-Hexanol } & 51 & 98.3 & 7.9 & 7.6 \\
\hline & 9340 & 95.6 & 9.0 & 8.9 \\
\hline \multirow[t]{2}{*}{ 2-Phenylethanol } & 1400 & 96.9 & 8.9 & 8.6 \\
\hline & 360,000 & 98.9 & 8.8 & 8.3 \\
\hline \multirow[t]{2}{*}{ 4-Terpineol } & 5 & 99.7 & 7.8 & 7.4 \\
\hline & 65.3 & 97.4 & 7.8 & 8.3 \\
\hline \multirow[t]{2}{*}{ Eugenol } & 0.05 & 98.6 & 6.7 & 7.4 \\
\hline & 5.6 & 96.7 & 6.4 & 9.8 \\
\hline \multirow[t]{2}{*}{ Hexanoic acid } & 8.73 & 94.9 & 5.6 & 6.7 \\
\hline & 78.6 & 93.4 & 6.6 & 7.6 \\
\hline \multirow[t]{2}{*}{ Octanoic acid } & 2744 & 93.1 & 10.9 & 11.3 \\
\hline & 197,200 & 92.5 & 11.1 & 11.4 \\
\hline \multirow[t]{2}{*}{ Decanoic acid } & 20.2 & 93.9 & 9.9 & 10.3 \\
\hline & 1608 & 93.2 & 8.7 & 9.4 \\
\hline \multirow[t]{2}{*}{ Dodecanoic acid } & 5.48 & 90.8 & 13.4 & 13.8 \\
\hline & 49.6 & 92.4 & 9.7 & 10.2 \\
\hline
\end{tabular}

a Relative standard deviation of assay done on the same day.

b Relative standard deviation of assay done on two different days. 
values from 0.948 to 0.942 . Noguerol-Pato et al. (2009) found $\mathrm{r}^{2}$ higher than 0.99 for terpenes, C-13 norisoprenoids, alcohols, esters and phenols. Antalick et al. (2010) investigated only esters and obtained $r^{2}$ that was also higher than 0.99 , while in this work $r^{2}$ ranged from 0.998 to 0.981 . However, Pozo-Bayon, Pueyo, Martin-Álvarez, and Polo (2001) showed lower $\mathrm{r}^{2}$ values, such as 0.96 for aldehydes and esters, while the $r^{2}$ of acids was in the range of 0.966 to 0.936 . Howard et al. (2005) reported that acids presented poor extraction and chromatographic behavior, besides being present in low concentrations in wines, and these were the reasons why they had not included fatty acids in their analytical method. On the other hand, Olivero and Trujillo (2011) developed a method for the determination of nine short-chain fatty acids (acetic, propionic, isobutyric, butyric, isovaleric, 2-methylbutyric, hexanoic, octanoic and decanoic acids) in wines using the HS/SPME-GC/ion trap MS and found $r^{2}>0.997$. In this case, the method was focused only on fatty acids and all conditions involved in extraction and analyses were optimized having acids as a goal, instead of having all the volatile compound classes as target analytes, as is the case in the present study.

The lower $r^{2}$ values were found for acids (octanoic, decanoic, dodecanoic acid) and this has probably happened because of chromatographic tailing due to their stronger interaction with the stationary phase. The calculated LOD and LOQ for each one of the 22 compounds are shown in Table 1 . The values of LOD ranged from $0.001 \mu \mathrm{g} \mathrm{L}^{-1}$ for ethyl isovalerate and hexanoic acid to $2.554 \mu \mathrm{g} \mathrm{L}^{-1}$ for ethyl 3-hydroxybutanoate. LOQ ranged from $0.003 \mu \mathrm{g} \mathrm{L}^{-1}$ for ethyl isovalerate and hexanoic acid to $7.582 \mu \mathrm{g} \mathrm{L}^{-1}$ for ethyl 3-hydroxy-butanoate. The LOQ and LOD values determined for all 22 compounds (Table 1 ) were found to be lower than the ones reported in other scientific works that have also employed HS-SPME-1D-GC/MS (Antalick et al., 2010; Howard et al., 2005; Noguerol-Pato et al., 2009; Perestrelo et al., 2006).

The precision test (repeatability and intermediate precision) has been performed in two different concentration levels (low and high) for each standard compound and results are shown in Table 3. These levels were used because they correspond to the lowest and the highest concentrations of each volatile compound that was used for the calibration curve. Repeatability ranged from $1.2 \%$ for ethyl decanoate to $13.4 \%$ for dodecanoic acid and intermediate precision ranged from $2.3 \%$ for ethyl 2-phenyl acetate to $13.8 \%$ for dodecanoic acid (Table 3 ). The only RSD values higher than $10 \%$ were found for octanoic acid and dodecanoic acid and the reason for that is probably the same mentioned for the lower $r^{2}$ found for carboxylic acids: its stronger interaction with the stationary phase that causes chromatographic tailing. Lower accuracies were also observed for acids.

Table 3 shows the accuracies for each one of the 22 standard compounds in two levels of concentration (low and high). The accuracy percent ranged from $92.4 \%$ for dodecanoic acid to $102.6 \%$ for ethyl octanoate. These accuracy results are in accord with data reported by other researchers that also used HS-SPME (DVB-CAR-PDMS) and GC/ MS to analyze wines (Antalick et al., 2010; Howard et al., 2005; Noguerol-Pato et al., 2009).

\subsection{Volatile compound quantification and odor activity value evaluation}

The concentration of the volatile compounds of the headspace of a Chardonnay wine is shown in Table 2 and is expressed in $\mu \mathrm{L} \mathrm{L}^{-1}$ as the average of five analytical replicates. OAV can be used to establish which compounds contribute to aroma because OAV calculation depends both on measured concentration and on the odor threshold of a specific compound in a certain matrix. Odor threshold and odor descriptor for the calculation of OAV of each tentatively identified compound were obtained from the scientific literature. Calculated $\mathrm{OAV}$ and literature references are given in Table 2. A volatile compound will contribute to the final wine aroma if its concentration is above its threshold perception (Guth, 1997; Li et al., 2008; Vilanova \& Martinez, 2007). A compound should present an odor activity value $>1$ in order to be perceived by human nose. Forty-seven compounds of Chardonnay wine showed $\mathrm{OAV}>1$ and this number corresponds to $19 \%$ of total identified compounds (Table 2). Among them, esters are the class that includes the larger number of odorant components (51\%), especially acetates and ethyl esters. The higher OAV values for ethyl esters are the ones of ethyl octanoate (\# 125 in Table 2, OAV $=130.0$, sweet, fruity or pear aroma (Peinado et al., 2004), ethyl hexanoate (\# 111 in Table 2, $\mathrm{OAV}=48.2$, fruity, green apple, brandy or wine-like aroma (Peinado et al., 2004) and ethyl butanoate (\# 104 in Table 2, OAV = 38.0, strawberry or apple aroma (Peinado et al., 2004) that are enzymatically produced during yeast fermentation and also during ethanolysis of acylCoA, that is formed during fatty acids synthesis or degradation. Their concentration is dependent on several factors, mainly: yeast strain, fermentation temperature, aeration degree, and sugar content (Etievant, 1991; Bakker \& Clarke, 2011).

Acetates (isoamyl acetate, $\mathrm{OAV}=9.6$ and butyl acetate, $\mathrm{OAV}=7.8$ ) also are among the compounds with higher OAV (Table 2) and are the result of the reaction of acetyl-CoA with alcohols and are formed from degradation of amino acids and carbohydrates (Etievant, 1991). In general, acetate and ethyl esters contribute to the fruity aroma of wine (Gurbuz et al., 2006; Peinado et al., 2004; Tao \& Zhang, 2010).

The acids that showed OAV $>1$ were octanoic, decanoic and hexanoic acid (OAV $=16.0,3.0$ and 1.5, \# 67, 70, 63 in Table 2, respectively). Volatile acids are produced during alcoholic fermentation and the contribution for the aroma depends on their concentration range in wine (Etievant, 1991). Shinohara (1985) showed that at concentrations of 4 to $10 \mathrm{mg} \mathrm{L}^{-1}, \mathrm{C}_{6}$ to $\mathrm{C}_{10}$ acids provide mild and pleasant aroma to wine. However, the impact of the presence of volatile acids may be negative when the concentration of these compounds is greater than $20 \mathrm{mg} \mathrm{L}^{-1}$ (Shinohara, 1985). In this study, only octanoic acid was found in a concentration that may negatively affect the wine aroma (160 $\left.\mathrm{mg} \mathrm{L}^{-1}\right)$.

Aldehydes and ketones may be formed by decarboxylation of acids carried by yeast, such as $\alpha$-keto acids including $\alpha$-ketolatic, $\alpha$ ketobutiric, $\alpha$-ketoisovaleric, $\alpha$-ketoisocaproic acids and others (Bakker \& Clarke, 2004). Nonanal (\# 86 in Table 2, OAV = 2.8), hexanal (\# 83 in Table 2, OAV = 2.1) and 2-phenylacetaldehyde (\# 91 in Table 2, OAV = 1.7) are among the most important aldehydes for wine aroma, whose odor descriptors are positive.

The ketone that showed higher OAV is 2,3-butanedione (\# 189 in Table 2 , OAV $=8.7$ ). This compound, also called diacetyl ketone, is important to the wine aroma and its contribution depends on its concentration in wine. Undesirable aroma can be perceived when the concentration of 2,3 butanedione is above $7.5 \mathrm{mg} \mathrm{L}^{-1}$. However, when present at concentrations below $4 \mathrm{mg} \mathrm{L}^{-1}$, it contributes positively with buttery or caramel aroma (Davis, Wibowo, Eschenbruch, Lee, \& Fleet, 1985). In the case of Chardonnay wines evaluated in this work, 2,3-butanedione was found in $0.18 \mathrm{mg} \mathrm{L}^{-1}$, which can result in pleasant odor perception.

The terpenes found in Chardonnay wines, in concentrations above odor threshold were: $p$-cymene (\# 210 in Table $2, \mathrm{OAV}=2.4$ ), linalool (\# 214 in Table 2, OAV = 1.9), $(E)$-nerolidol $(\# 222$ in Table $2, \mathrm{OAV}=$ 1.6), 3-carene (\# 209 in Table 2, OAV = 1.6), $\alpha$-citronellol (\# 219 in Table $2, \mathrm{OAV}=1.2),(E)$-farnesol $(\# 223$ in Table $2, \mathrm{OAV}=1.2)$, and $\alpha$-terpineol (\# 217 in Table 2, OAV $=1.1$ ). These compounds tend to contribute positive floral aromas to wine aroma and their odor description is reported in Table 2. Terpene and C13-norisoprenoids are part of the grape varietal aroma and may undergo fermentation without substantial changes. They may be found in grape skin and maceration is an essential step for the transference of these compounds to the grape must (Bakker \& Clarke, 2004).

$\beta$-Damascenone (\# 206 in Table 2, OAV $=20$ ) is the only C13norisoprenoid with OAV able to positively contribute to the aroma of the wine. However, this compound can synergistically change the aroma of other compounds. In hydroalcoholic solution, $\beta$-damascenone has enhanced fruity notes of ethyl cinnamate and caproate and masked 

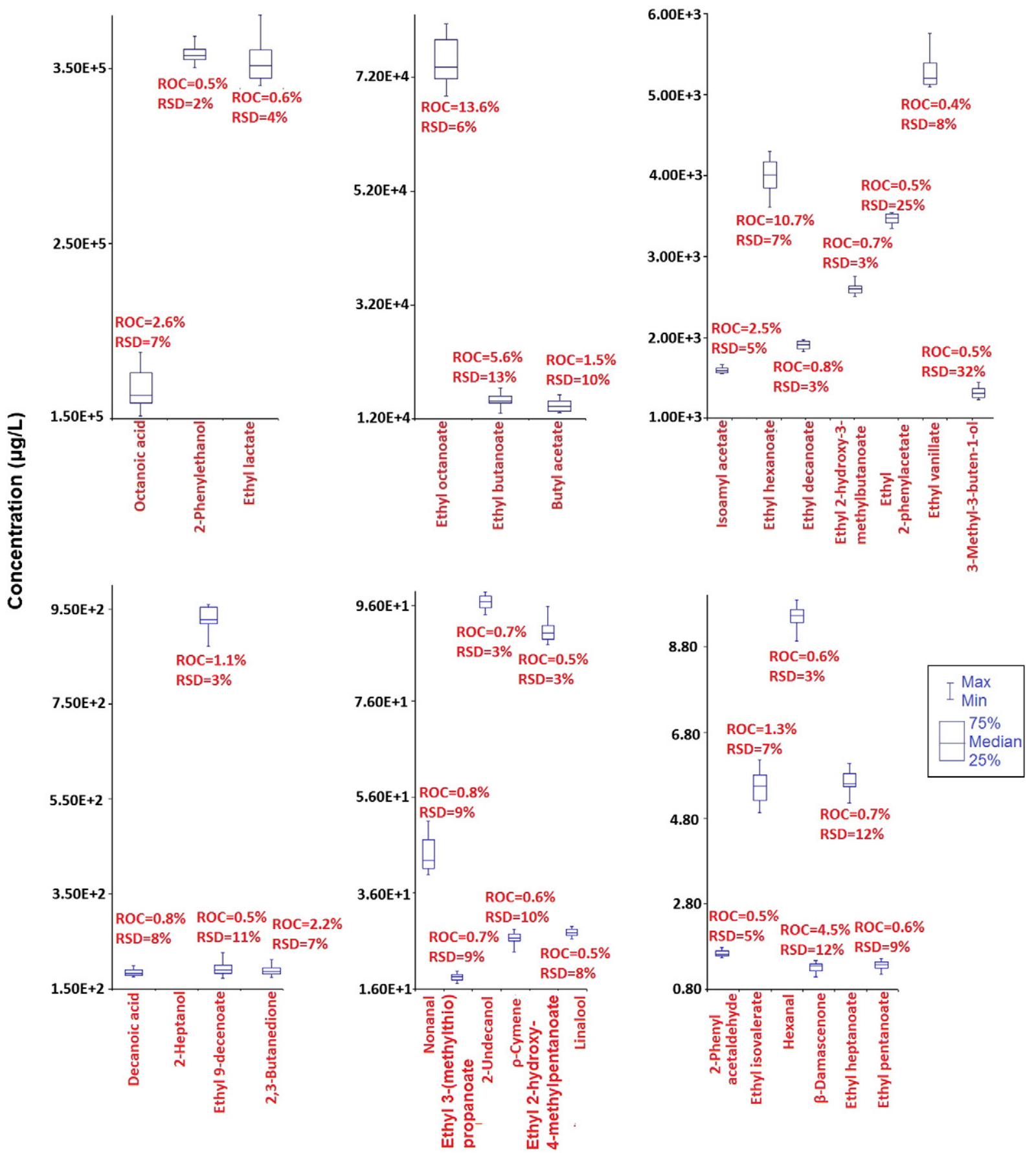

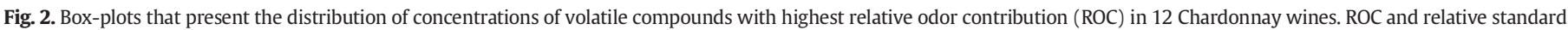
deviation (RSD) are shown for each compound.

the herbaceous aroma of 3-isobutyl-2-methoxypyrazine (IBMP) (Pineau, Barbe, Leeuwen, \& Dubourdieu, 2007).

The contribution of each volatile compound with OAV $>1$ to wine aroma can be evaluated qualitatively by means of its associate descriptor, and quantitatively by means of its OAV or ROC (Table 2). In Fig. 2, the quantified compounds were grouped according to their concentration range (in the following order: $10^{5}, 10^{4}, 10^{3}, 10^{2}, 10$ and $<10 \mu \mathrm{g} \mathrm{L}^{-1}$ ). This approach was necessary due to the great variability of concentration among different tentatively identified compounds, ranging from 0.89 to more than $37,000 \mu \mathrm{g} \mathrm{L}-1$. The box plot of Fig. 2 shows 29 compounds with the highest OAV $(>1.7)$ that were quantified in twelve Chardonnay wine samples of the Serra Gaúcha region, including those found in the order of $10^{5} \mu \mathrm{g} \mathrm{L}{ }^{-1}$ : octanoic acid $(\mathrm{OAV}=16$, \# 67 in
Table 2), 2-phenylethanol (OAV = 1.8, \# 43), ethyl lactate (OAV = 2.3, \# 117); $10^{4} \mu \mathrm{g} \mathrm{L}^{-1}$ : ethyl octanoate (OAV $=130$, \# 125), ethyl butanoate $(\mathrm{OAV}=38, \# 104)$, butyl acetate $(\mathrm{OAV}=7.8, \# 107)$; $10^{3} \mu \mathrm{g} \mathrm{L}^{-1}$ : isoamyl acetate ( $\left.\mathrm{OAV}=9.6, \# 108\right)$, ethyl hexanoate $(\mathrm{OAV}=48.2$, \# 111), ethyl decanoate $(\mathrm{OAV}=3.9$, \# 147), ethyl 2-hydroxy-3-methylbutanoate $(\mathrm{OAV}=2.6$, \# 119), ethyl 2phenylacetate $(\mathrm{OAV}=1.9$, \# 164), ethyl vanillate, 3-methyl-3-buten-1ol; $10^{2} \mu \mathrm{g} \mathrm{L}^{-1}$ : decanoid acid $(\mathrm{OAV}=3$; \# 70), heptanol $(\mathrm{OAV}=4.7$, \# 9), ethyl 9-decenoate (OAV $=1.9$, \# 156), 2,3-butanedione $(\mathrm{OAV}=8.7, \# 189) ; 10 \mu \mathrm{g} \mathrm{L}{ }^{-1}$ : nonanal $(\mathrm{OAV}=2.8$, \# 86), ethyl 3-(methylthio)propanoate (OAV $=2.7, \# 236)$, 2-undecanol $(\mathrm{OAV}=2.4, \# 36), \rho$-cymene $(\mathrm{OAV}=2.4$, \# 210), ethyl 2-hydroxy-4methyl-pentanoate $(\mathrm{OAV}=2.1$, \# 106), linalool $(\mathrm{OAV}=1.9$, \# 214); 

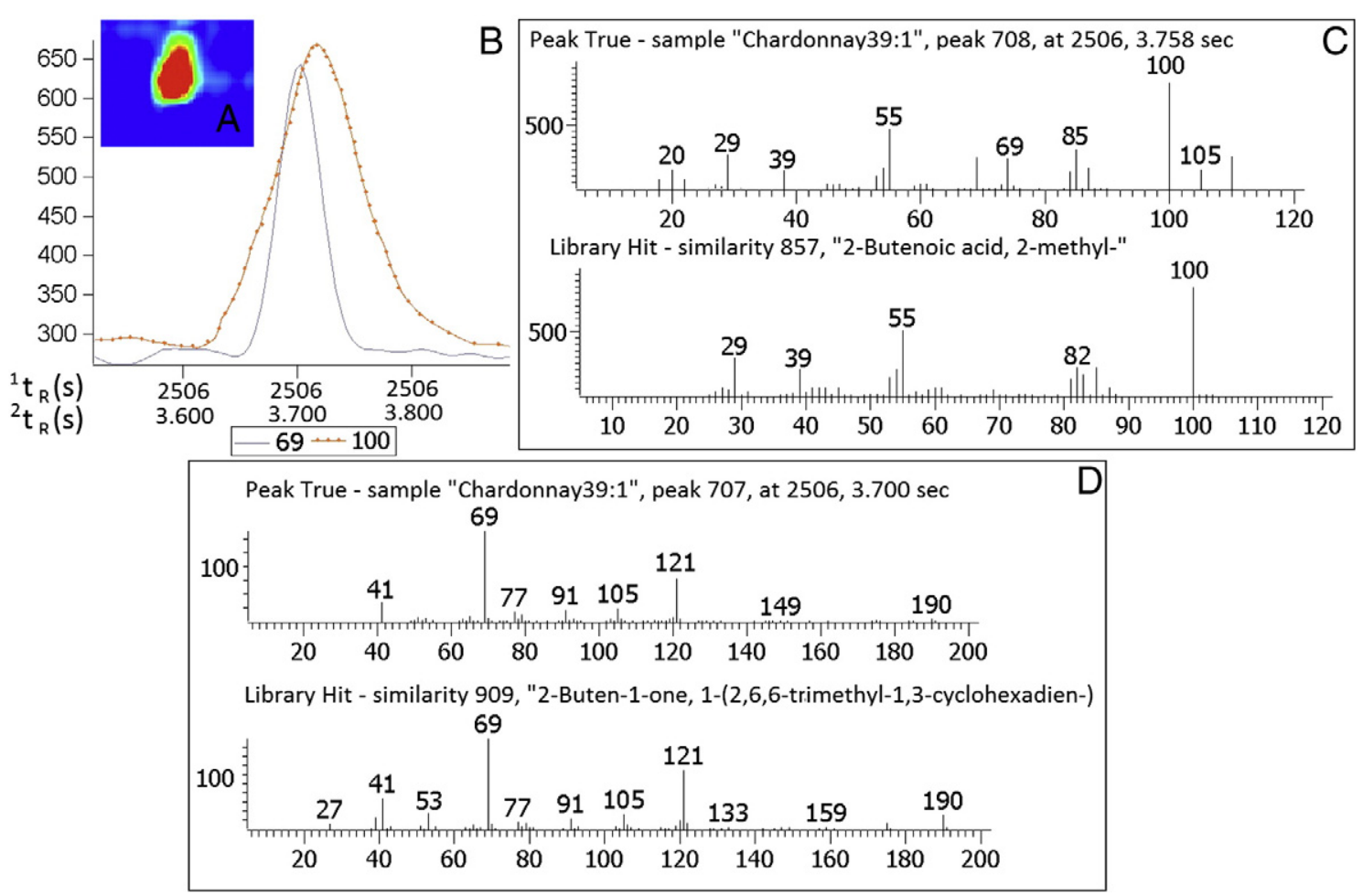

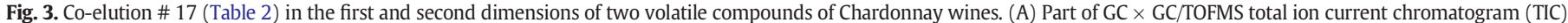

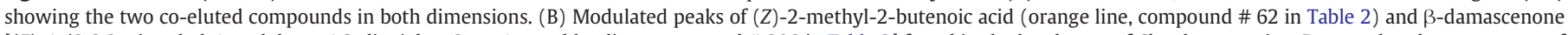

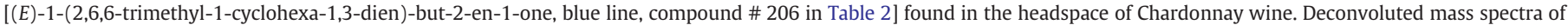
(C) (Z)-2-methyl-2-butenoic acid and (D) $\beta$-damascenone.

$<10 \mu \mathrm{g} \mathrm{L}^{-1}$ : 2-phenylacetaldehyde (OAV $=1.7$, \# 91), ethyl isovalerate $(\mathrm{OAV}=5.3, \# 105)$, hexanal $(\mathrm{OAV}=2.1, \# 83), \beta$-damascenone $(\mathrm{OAV}=$ 20 , \# 206), ethyl heptanoate $(O A V=2.5$, \# 114) and ethyl pentanoate $(\mathrm{OAV}=2.1$, \# 106). The box-plot provides information on dispersion and asymmetry of the quantitative data. The top and bottom rows of the box indicate that $75 \%$ and $25 \%$, respectively, of the samples have lower concentration than the value expressed by these bars.

The ROC, that represents the contribution percentage of the each volatile compound to aroma, is also shown in Fig. 2. Ethyl octanoate is the compound that showed the highest contribution to aroma $(\mathrm{ROC}=13.6 \%)$, followed by ethyl hexanoate $(\mathrm{ROC}=10.7 \%)$ and ethyl butanoate $(\mathrm{ROC}=5.6 \%)$. Ethyl octanoate and ethyl hexanoate are also the compounds with more expressive contribution to the aroma of Cabernet Sauvignon, Cabernet Gernischet and Chardonnay wines from China (Jiang \& Zhang, 2010). However, the contributions of these compounds to the aroma of Chinese wines were 92.9 and 93.3\%, respectively. Several aspects may explain differences between the aroma of Brazilian and Chinese Chardonnay, as for example climate, enological practices, etc. Furthermore, extraction and analysis of the Chinese wines were performed with HS-SPME-GC/MS and only twelve among the 42 tentatively identified volatile compounds showed $\mathrm{OAV}>1$. In the present work a higher number of compounds (243) were tentatively identified using HS-SPME-GC $\times$ GC/TOFMS and 47 of them had OAV $>1$. Then, as a higher number of compounds contribute to the flavor of the Serra Gaucha Chardonnay, the percentage contribution of each compound to the overall aroma, expressed by the ROC, is reduced.

ROC relative standard deviation (RSD) for each compound in twelve Chardonnay wines is shown in Fig. 2. The variation in the concentration of the majority of the volatile compounds responsible for the aroma of the twelve Chardonnay wines was less than $10 \%$ for $83 \%$ of the compounds. These data show that these twelve Chardonnays produced in the Serra Gaucha region presented a reasonable similarity among them regarding aroma. Ethyl 2-phenylacetate $(\mathrm{RSD}=25 \%)$ and 3-methyl-3-buten-1-ol were the compounds with the highest RSD for ROC values. However their contribution to aroma is not among the most expressive, as their ROC values are low ( $0.5 \%$ for each compound).

$\beta$-Damascenone was the fourth compound with highest ROC $($ ROC $=4.5 \%)$ (Fig. 2). This C13-norisoprenoid (\# 206 in Table 2) co-eluted with (Z)-2-methyl-2-butenoic acid (\# 62 in Table 2) in both first and second dimension (Fig. 3). Fig. 3A and B shows the superimposed chromatographic peaks and Fig. 3 C and D, the mass spectra of the two compounds compared with their mass spectra in the NIST library. Spectral deconvolution based on mass spectra differences was quite useful in this case, as chromatographic selectivity was not enough to achieve separation, even though two different stationary phases have been employed for analysis. The odor of $(Z)$-2-methyl-2-butenoic acid is described as spicy, pungent (Lee et al., 2008), while $\beta$-damascenone can contribute to sweet or honey odor (Gurbuz et al., 2006). Correct separation and identification of these compounds are crucial for a fair characterization of important aroma characteristics and this illustrates the importance of spectral deconvolution.

\section{Conclusions}

The well known higher capacity of GC $\times$ GC/TOFMS for the analysis of complex samples proved to be appropriate for this first quantitative analysis of volatile compounds of Chardonnay wines, as separation of first dimension co-elutions were achieved through the selectivity of the second dimension column and also through spectral deconvolution, rendering an adequate method validation for a detailed analysis. Co-elutions in ${ }^{1} \mathrm{D}$ point to difficulties that might arise whenever only 1D-GC/MS is employed, possibly resulting in insufficient chromatographic separation and consequently incorrect identification and quantitative results. A higher number of compounds with $\mathrm{OAV}>1$ than are usually found by $1 \mathrm{D}-\mathrm{GC} / \mathrm{MS}$ were verified and this also might be attributed to the higher efficiency of GC $\times$ GC/TOFMS. 
The combination of GC $\times$ GC/TOFMS for qualitative and quantitative analyses of Chardonnay wines along with the OAV and ROC approaches has been performed for the first time and has been shown to be advantageous, as it allowed a fast overview of the linking between concentration, odor descriptors, and odorant impact for each volatile compound, resulting in the discovery of the most important compounds for the aroma of Chardonnay wines and their concentrations. A detailed quantitative analysis combined with OAV and ROC determination for a varietal wine, followed by the same procedure focused only on the most important odorant compounds for a higher number of wine samples of the same varietal wine paves the way to facilitate the implementation of meaningful quality control for the wine industry. Future work for a better characterization of the aromatic profile of wines, using olfactometric detector with 1D-GC and/or GC $\times$ GC will be important for a more detailed description of the contribution of each compound to the aroma. Furthermore, the effect of synergistic interactions and sensory evaluation by trained panelists should be considered to complement these studies of the aroma of Brazilian wines.

\section{Acknowledgments}

The authors thank the Conselho Nacional de Desenvolvimento Científico e Tecnológico (CNPq), the Coordenação de Aperfeiçoamento de Pessoal de Nível Superior (CAPES), and the Fundação de Amparo a Pesquisa do Estado do Rio Grande do Sul (FAPERGS) for financial support and scholarships. Leila Falcão is also especially acknowledged for contributing the standard compounds for this research project.

\section{References}

Annan, N. T., Poll, L., Plahar, W. A., \& Jakobsen, M. (2003). Aroma characteristics of spontaneously fermented Ghanaian maize dough for kenkey. European Food Research and Technology, 217, 53-60.

Antalick, G., Perello, M. C., \& Revel, G. (2010). Development, validation and application of a specific method for the quantitative determination of wine esters by headspace-solid-phase microextraction-gas chromatography-mass spectrometry. Food Chemistry, 121, 1236-1245.

Bakker, J., \& Clarke, R. (2011). Wine: Flavour chemistry (2nd ed.). Oxford: Wiley-Blackwell.

Bendahou, M., Muselli, A., Grignon-Dubois, M., Benyoucef, M., Desiobert, J. M., Bernardini, A. F., et al. (2008). Antimicrobial activity and chemical composition of Origanum glandulosum Desf. essential oil and extract obtained by microwave extraction: Comparison with hydrodistillation. Food Chemistry, 106, 132-139.

Bianchi, F., Careri, M., Mangia, A., \& Musci, M. (2007). Retention indices in the analysis of food aroma volatile compounds in temperature-programmed gas chromatography: database creation and evaluation of precision and robustness. Journal of Separation Science, 30, 563-572.

Boonbumrung, S., Tamura, H., Mookdasanit, J., Nakamoto, H., Ishihraa, M., Yoshizawa, T. et al. (2001). Characteristic aroma components of the volatile oil of yellow keaw mango fruits determined by limited odor unit method. Food Science and Technology Research, 7, 200-206.

Bordiga, M., Rinaldi, M., Locatelli, M., Piana, G., Travaglia, F., Coïsson, J. D., \& Arlorio, M. (2013). Characterization of Muscat wines aroma evolution using comprehensive gas chromatography followed by a post-analytic approach to 2D contour plots comparison. Food Chemistry, 140, 57-67.

Brunton, N. P., Cronin, D. A., \& Monahan, F. J. (2002). Volatile components associated with freshly cooked and oxidized off-flavours in turkey breast meat. Flavour and Fragrance Journal, 17, 327-334.

Buttery, R. G., Orts, G. W. J., Takeoka, G. R., \& Nam, Y. (1999). Volatile flavor components of rice cakes. Journal of Agricultural and Food Chemistry, 47, 4353-4356.

Capone, S., Tufariello, M., \& Siciliano, P. (2013). Analytical characterisation of Negroamaro red wines by "Aroma Wheels". Food Chemistry, 141, 2906-2915

Chastrette, M., Cretin, D., \& El Aidi, C. (1996). Structure-odor relationships: Using neura networks in the estimation of camphoraceous or fruity odors and olfactory thresholds of aliphatic alcohols. Journal of Chemical Information and Computer Sciences, 36 108-113.

Choi, H. S. (2003). Character impact odorants of Citrus hallabong (C. unshiu Marcov C. sinensis Osbeck) $\times$ C. reticulata Blanco cold-pressed peel oil. Journal of Agricultura and Food Chemistry, 51, 2687-2692.

Chyau, C. C., Chen, S. Y., \& Wu, C. M. (1992). Differences of volatile and nonvolatile constituents between mature and ripe guava (Psidium guajava Linn) fruits. Journal of Agricultural and Food Chemistry, 40, 846-849.

Condurso, C., Verzera, A., Romeo, V., Ziino, M., \& Conte, F. (2008). Solid-phase microextraction and gas chromatography mass spectrometry analysis of dairy product volatiles for the determination of shelf-life. International Dairy Journal, 18 819-825.
Cullere, L., Escudero, A., Cacho, J., \& Ferreira, V. (2004). Gas chromatography olfactometry and chemical quantitative study of the aroma of six premium quality Spanish aged red wines. Journal of Agricultural and Food Chemistry, 52, 1653-1660.

Davis, C. R., Wibowo, D., Eschenbruch, R., Lee, T. H., \& Fleet, G. H. (1985). Practical implications of malolactic fermentation: A review. American Journal of Enology and Viticulture, 36, 290-301.

Dugo, G., Franchina, F. A., Scandinaro, M. R., Bonaccorsi, I., Cicero, N., Tranchida, P. Q., \& Mondello, L. (2014). Elucidation of the volatile composition of Marsala wines by using comprehensive two-dimensional gas chromatography. Food Chemistry, 142, 262-268.

Etiévant, P. X. (1991). Wine. In H. Maarse (Ed.), Volatile compounds in foods and beverages (pp. 483-546). New York: Dekker

Fan, Y., \& Qian, M. C. (2006). Characterization of aroma compounds of Chinese "Wuliangye" and "Jiannanchun" liquors by aroma extract dilution analysis. Journal of Agricultural and Food Chemistry, 54, 2695-2704.

Fan, W., Xu, Y., Jiang, W., \& Li, J. (2010). Identification and quantification of impact aroma compounds in 4 nonfloral Vitis vinifera varieties grapes. Journal of Food Science, 75, 81-88.

Ferrari, G., Lablanquie, O., Cantagrel, R., Ledauphin, H., Payot, T., Fournier, N., et al. (2004). Journal of Agricultural and Food Chemistry, 52, 5670-5676

Ferreira, V., Lopez, R., \& Cacho, J. F. (2000). Quantitative determination of the odorants of young red wines from different grape varieties. Journal of the Science of Food and Agriculture, 80, 1659-1667.

Frohlich, O., Duque, C., \& Schreier, P. (1989). Volatile constituents of curuba (Passiflora mollissima) fruit. Journal of Agricultural and Food Chemistry, 37, 421-425.

Fu, S. G., Yoon, Y., \& Bazemore, R. (2002). Aroma-active components in fermented bamboo shoots. Journal of Agricultural and Food Chemistry, 50, 549-554.

Garcia, C. V., Quek, S. Y., Stevenson, R. J., \& Winz, R. A. (2011). Characterization of the bound volatile extract from baby kiwi (Actinidia arguta). Journal of Agricultural and Food Chemistry, 59, 8358-8365.

Garcia-Carpintero, E. G., Sanchez-Palomo, E., Gallego, M. A. G., \& Gonzalez-Viñas, M. A. (2011). Volatile and sensory characterization of red wines from cv. Moravia Agria minority grape variety cultivated in La Mancha region over five consecutive vintages. Food Research International, 44, 1549-1560.

Giri, A., Osako, K., \& Ohshima, T. (2010). Identification and characterisation of headspace volatiles of fish miso, a Japanese fish meat based fermented paste, with special emphasis on effect of fish species and meat washing. Food Chemistry, 120, 621-631.

Guo, L., Wu, J. Z., Han, T., Cao, T., Rahman, K., \& Qin, L. P. (2008). Chemical composition, antifungal and antitumor properties of ether extracts of Scapania verrucosa Heeg. and its endophytic fungus Chaetomium fusiforme. Molecules, 13, 2114-2125.

Gurbuz, O., Rouseff, J. M., \& Rouseff, R. L. (2006). Comparison of aroma volatiles in commercial Merlot and Cabernet Sauvignon wines using gas chromatography olfactometry and gas chromatography mass spectrometry. Journal of Agricultural Food Chemistry, 54, 3990-3996.

Guth, H. (1997). Quantification and sensory studies of character impact odorants of different white varieties. Journal of Agricultural and Food Chemistry, 45, 3027-3032.

Howard, K. L., Mike, J. H., \& Riesen, R. (2005). Validation of a solid-phase microextraction method for headspace analysis of wine aroma components. American Journal of Enology and Viticulture, 56, 37-45.

ICH - International Conference on Harmonization of Technical Requirements for Registration of Pharmaceuticals for Human Use (1996). Q2B validation of analytical procedures: Methodology.

Iwaoka, W., Hagi, Y., Umano, K., \& Shibamoto, T. (1994). Volatile chemicals identified in fresh and cooked breadfruit. Journal of Agricultural and Food Chemistry, 42, 975-976.

Jackson, R. S. (2008). Wine science: Principles and applications. Boston: Elsevier.

Jaffré, J., Valentin, D., Meunier, J. M., Siliani, A., Bertuccioli, M., \& Le Fur, Y. (2011). The Chardonnay wine olfactory concept revisited: A stable core of volatile compounds, and fuzzy boundaries. Food Research International, 44, 456-464.

Jang, H., Ka, M., \& Lee, K. (2008). Antioxidant activity and characterization of volatile extracts of Capsicum annuum L. and Allium spp. Flavour and Fragrance Journal, 23, $178-184$

Jiang, B., \& Zhang, Z. (2010). Volatile compounds of young wines from Cabernet Sauvignon, Cabernet Gernischet and Chardonnay varieties grown in the Loess Plateau Region of China. Molecules, 15, 9184-9196.

Juan, S. F., Cacho, J., Ferreira, V., \& Escudero, A. (2012). Aroma chemical composition of red wines from different price categories and its relationship to quality. Journal of Agricultural and Food Chemistry, 60, 5045-5056.

Kourkoutas, D., Elmore, J. S., \& Mottram, D. S. (2006). Comparison of the volatile compositions and flavour properties of cantaloupe, Galia and honeydew muskmelons. Food Chemistry, 97, 95-102.

Kumazawa, K., Itobe, T., Nishimura, O., \& Hamaguchi, T. (2008). A new approach to estimate the in-mouth release characteristics of odorants in chewing gum. Food Science and Technology Research, 14, 269-276.

Ledauphin, J., Saint-Clair, J. F., Lablanquie, O., Guichard, H., Founier, N., Guichard, D., et al. (2004). Identification of trace volatile compounds in freshly distilled calvados and cognac using preparative separations coupled with gas chromatography-mass spectrometry. Journal of Agricultural and Food Chemistry, 52, 5124-5134.

Lee, K., \& Shibamoto, T. (2000). Antioxidant properties of aroma compounds isolated from soybeans and mung beans. Journal of Agricultural and Food Chemistry, 48, 4290-4293.

Lee, G. H., Shin, Y., \& Oh, M. J. (2008). Aroma-active components of Lycii fructus (kukija). Journal of Food Science, 73, 500-505.

Lee, S. J., Umano, K., Shibamoto, T., \& Lee, K. G. (2005). Identification of volatile components in basil (Ocimum basilicum L.) and thyme leaves (Thymus vulgaris L.) and their antioxidant properties. Food Chemistry, 91, 131-137. 
Li, H., Tao, Y. S., Wang, H., \& Zhang, L. (2008). Impact odorants of Chardonnay dry white wine from Changli County (China). European Food Research and Technology, 227, 287-292.

Liu, X., Jin, Q., Liu, Y., Hunag, J., Wang, X., Mao, W., et al. (2011). Changes in volatile compounds of peanut oil during the roasting process for production of aromatic roasted peanut oil. Journal of Food Science, 76, 404-412.

Lytra, G., Tempere, S., Revel, G., \& Barbe, J. C. (2012). Distribution and organoleptic impact of ethyl 2-hydroxy-4-methylpentanoate enantiomers in wine. Journal of Agricultural and Food Chemistry, 60, 1503-1509.

Mahajan, S. S., Goddik, L., \& Qian, M. C. (2004). Aroma compounds in sweet whey powder. Journal of Dairy Science, 87, 4057-4063.

Malherbe, S., Menichelli, E., du Toit, M., Tredoux, A., Muller, N., Næs, T., et al. (2013). The relationships between consumer liking, sensory and chemical attributes of Vitis vinifera L. cv. Pinotage wines elaborated with different Oenococcus oeni starter cultures. Journal of the Science of Food and Agriculture, 93, 2829-2840.

Marriott, P. J., \& Shellie, R. (2002). Principles and applications of comprehensive two-dimensional gas chromatography. Trends in Analytical Chemistry, 21, 573-583.

Martínez-Pinilla, O., Guadalupe, Z., Ayestarán, B., Pérez-Magariño, S., \& Ortega-Heras, M. (2013). Characterization of volatile compounds and olfactory profile of red minority varietal wines from La Rioja. Journal of the Science of Food and Agriculture, 93, 3720-3729.

Mayorga, H., Knapp, H., Winterhalter, P., \& Duque, C. (2001). Glycosidically bound flavor compounds of cape gooseberry (Physalis peruviana L.). Journal of Agricultural and Food Chemistry, 49, 1904-1908.

Meret, M., Brat, P., Mertz, C., Lebrun, M., \& Guenata, Z. (2011). Contribution to aroma potential of Andean blackberry (Rubus glaucus Benth.). Food Research International, $44,54-60$.

Morales, A. L., \& Duque, C. (2002). Free and glycosidically bound volatiles in the mammee apple (Mammea americana) fruit. European Food Research and Technology, 215. 221-226.

Niu, Y., Zhang, X., Xiao, Z., Song, S., Eric, K., Jia, C., et al. (2011). Characterization of odor-active compounds of various cherry wines by gas chromatography-mass spectrometry, gas chromatography-olfactometry and their correlation with sensory attributes. Journal of Chromatography B, 879, 2287-2293.

Noguerol-Pato, R., González-Barreiro, C., Cancho-Grande, B., \& Simal-Gándara, J. (2009). Quantitative determination and characterisation of the main odourants of Mencía monovarietal red wines. Food Chemistry, 117, 473-484.

Olivero, S. J. P., \& Trujillo, J. P. P. (2011). A new method for the determination of short-chain fatty acids from the aliphatic series in wines by headspace solid-phase microextraction-gas chromatography-ion trap mass spectrometry. Analytica Chimica Acta, 696, 59-66.

Peinado, R. A., Moreno, J. A., Muñoz, D., Medina, M., \& Moreno, J. (2004). Gas chromatographic quantification of major volatile compounds and polyols in wine by direct injection. Journal of Agricultural and Food Chemistry, 52, 6389-6393.

Peng, C. T., Yang, Z. C., \& Ding, S. F. (1991). Prediction of retention indexes. II. Structure-retention index relationship on polar columns. Journal of Chromatography, 586, 85-112.

Perestrelo, R., Barros, A. S., Câmara, J. S., \& Rocha, S. M. (2011). In-depth search focused on furans, lactones, volatile phenols, and acetals as potential age markers of Madeira wines by comprehensive two-dimensional gas chromatography with time-of-flight mass spectrometry combined with solid phase microextraction. Journal of Agricultural and Food Chemistry, 59, 3186-3204.

Perestrelo, R., Fernandes, A., Albuquerque, F. F., Marques, J. C., \& Câmara, J. S. (2006). Analytical characterization of the aroma of Tinta Negra Mole red wine: Identification of the main odorants compounds. Analytica Chimica Acta, 563, 154-164.

Pineau, B., Barbe, J. C., Leeuwen, C. V., \& Dubourdieu, D. (2007). Which impact for B-damascenone on red wines aroma? Journal of Agricultural and Food Chemistry, 55, 4103-4108.

Pino, J. A., \& Quijano, C. E. (2012). Study of the volatile compounds from plum (Prunus domestica L. cv. Horvin) and estimation of their contribution to the fruit aroma. Ciência e Tecnologia de Alimentos, 32, 76-82.

Pozo-Bayon, M. A., Pueyo, E., Martin-Álvarez, P. J., \& Polo, M. C. (2001). Polydimethylsiloxane solid-phase microextraction-gas chromatography method for the analysis of volatile compounds in wines. Journal of Chromatography A, 922, 267-275.

Pozo-Bayon, M. A., Ruiz-Rodriguez, A., Pernin, K., \& Cayot, N. (2007). Influence of eggs on the aroma composition of a sponge cake and on the aroma release in model studies on flavored sponge cakes. Journal of Agricultural and Food Chemistry, 55, 1418-1426.

Qian, M., \& Wang, Y. (2005). Seasonal variation of volatile composition and odor activity value of 'Marion' (Rubus spp. hyb) and 'Thornless evergreen' ( $R$. laciniatus L.) blackberries. Journal of Food Science, 70, C13-C20.

Robinson, A. L., Boss, P. K., Heymann, H., Solomon, P. S., \& Trengove, R. D. (2011). Influence of yeast strain, canopy management, and site on the volatile composition and sensory attributes of Cabernet Sauvignon wines from Western Australia. Journal of Agricultural and Food Chemistry, 59, 3273-3284.

Ryona, I., Pan, B. S., \& Sacks, G. L. (2009). Rapid measurement of 3-alkyl-2methoxypyrazine content of winegrapes to predict levels in resultant wines. Journal of Agricultural and Food Chemistry, 57, 8250-8257.

Saidana, D., Mahjoub, S., Boussaada, O., Chriaa, J., Mahjoub, M. A., Cheraif, I., et al. (2008). Antibacterial and antifungal activities of the essential oils of two salt cedar species from Tunisia. Journal of the American Oil Chemists' Society, 85, 817-826.

Samappito, S., \& Butkhup, L. (2010). Effect of skin contact treatments on the aroma profile and chemical components of mulberry (Morus alba Linn.) wines. African Journal of Food Science, 4, 52-61.

Sampaio, K. L., Garruti, D. S., Franco, B. R. B., Janzantti, N. S., \& Da Silva, M. A. A. P. (2011). Aroma volatiles recovered in the water phase of cashew apple (Anacardium occidentale L.) juice during concentration. Journal of the Science of Food and Agriculture, 91, 1801-1809.

Sanz, C., Ansorena, D., Bello, J., \& Cid, C. (2001). Optimizing headspace temperature and time sampling for identification of volatile compounds in ground roasted Arabica coffee. Journal of Agricultural and Food Chemistry, 49, 1364-1369.

Selli, S., Bagatar, B., Sen, K., \& Kelebek, H. (2001). Evaluation of differences in the aroma composition of free-run and pressed neutral grape juices obtained from Emir (Vitis vinifera L.). Chemistry \& Biodiversity, 8, 1776-1782.

Selli, S., Canbas, A., Cabaroglu, T., Erten, H., \& Gunata, Z. (2006). Aroma components of cv. Muscat of Bornova wines and influence of skin contact treatment. Food Chemistry, 94 319-326.

Selli, S., \& Cayhan, G. G. (2009). Analysis of volatile compounds of wild gilthead sea bream (Sparus aurata) by simultaneous distillation-extraction (SDE) and GC-MS Microchemical Journal, 93, 232-235.

Shinohara, T. (1985). Gas chromatographic analysis of volatile fatty acids in wines. Agricultural and Biological Chemistry, 49, 2211-2212.

Simon, B. F., Esteruelas, E., Munoz, A. M., Cadahia, E., \& Sanz, M. (2009). Volatile compounds in acacia, chestnut, cherry, ash, and oak woods, with a view to their use in cooperage. Journal of Agricultural and Food Chemistry, 57, 3217-3227.

Takahashi, T., Mizuib, K., \& Miyazawa, M. (2010). Volatile compounds with characteristic odour in moso-bamboo stems (Phyllostachys pubescens Mazel ex Houz. De ehaie) Phytochemical Analysis, 21, 489-495.

Takeoka, G. R., Flath, R. A., Mon, T. R., Teranishi, R., \& Guentert, M. (1990). Volatile constituents of apricot (Prunus armeniaca). Journal of Agricultural and Food Chemistry, 38 471-477.

Tao, Y., \& Li, H. (2009). Active volatiles of Cabernet Sauvignon wine from Changli County. Natural Science, 1, 176-182.

Tao, Y., Li, H., Wang, H., \& Zhang, L. (2008). Volatile compounds of young Cabernet Sauvignon red wine from Changli County (China). Journal of Food Composition and Analysis, 21, 689-694.

Tao, Y., \& Zhang, L. (2010). Intensity prediction of typical aroma characters of cabernet sauvignon wine in Changli County (China). LWT - Food Science and Technology, 43, 1550-1556.

Umano, K., Hagi, Y., Nakahara, K., Shoji, A., \& Shibamoto, T. (1992). Volatile constituents of green and ripened pineapple (Ananas comosus [L.] Merr.). Journal of Agricultural and Food Chemistry, 40, 599-603.

Varlet, V., Prost, C., \& Serot, T. (2007). Volatile aldehydes in smoked fish: Analysis methods, occurrence and mechanisms of formation. Food Chemistry, 105, 1536.

Vekiari, S. A., Oreopoulou, V., Kourkoutas, Y., Kamoun, N., Msallem, M., Psimouli, V., et al. (2010). Characterization and seasonal variation of the quality of virgin olive oil of the Throumbolia and Koroneiki varieties from Southern Greece. Grasas Y Aceites, 61, 221-231.

Verzera, A., Ziino, M., Scacco, A., Lanza, C. M., Mazzaglia, A., Romeo, V., et al. (2008) Volatile compound and sensory analysis for the characterization of an Italian white wine from "Inzolia" grapes. Food Analytical Methods, 1, 144-151.

Vilanova, M., Genisheva, Z., Masa, A., \& Oliveira, J. M. (2010). Correlation between volatile composition and sensory properties in Spanish Albariño wines. Microchemical Journal, 95, 240-246.

Vilanova, M., \& Martinez, C. (2007). First study of determination of aromatic compounds of red wine from Vitis vinifera CV. Castanal grown in Galicia (NW Spain). European Food Research and Technology, 224, 431-436.

von Muhlen, C., Zini, C. A., Caramao, E. B., \& Marriott, P. J. (2008). Comparative study of Eucalyptus dunnii volatile oil composition using retention indices and comprehensive two-dimensional gas chromatography coupled to time-of-flight and quadrupole mass spectrometry. Journal of Chromatography A, 1200, 34-42.

Watcharananun, W., Cadwallader, K. R., Huangrak, K., Kim, H., \& Lorjaroenphon, Y. (2009). Identification of predominant odorants in Thai desserts flavored by smoking with "Tian Op", a traditional Thai scented candle. Journal of Agricultural and Food Chemistry, 57, 996-1005.

Weckerle, B., Bastl-Borrmann, R., Richling, E., Hor, K., Ruff, C., \& Schreier, P. (2001) Cactus pear (Opuntia ficus indica) flavour constituents - chiral evaluation (MDGC-MS) and isotope ratio (HRGC-IRMS) analysis. Flavour and Fragrance Journal, 16, 360-363.

Weldegergis, B. T., de Villiers, A., McNeish, C., Seethapathy, S., Mostafa, A., Gorecki, T., et al. (2011). Characterization of volatile components of Pinotage wines using comprehensive two-dimensional gas chromatography coupled to time-of-flight mass spectrometry (GC $\times$ GC-TOFMS). Food Chemistry, 129, 188-199.

Welke, J. E., Manfroi, V., Zanus, M., Lazarotto, M., \& Zini, C. A. (2012). Characterization of the volatile profile of Brazilian Merlot wines through comprehensive two dimensional gas chromatography time-of-flight mass spectrometric detection. Journal of Chromatography A, 1226, 124-139.

Welke, J. E., Manfroi, V., Zanus, M., Lazarotto, M., \& Zini, C. A. (2013). Differentiation of wines according to grape variety using multivariate analysis of comprehensive two-dimensional gas chromatography with time-of-flight mass spectrometric detection data. Food Chemistry, 141, 3897-3905.

Welke, J. E., Zanus, M., Lazarotto, M., Pulgati, F. H., \& Zini, C. A. (2014). Comparison of volatile profiles of base wines and their corresponding sparkling wines through comprehensive two dimensional gas chromatography time-of-flight mass spectrometric detection. (submitted for publication).

Welke, J. E., Zanus, M., Lazarotto, M., Schmitt, K. G., \& Zini, C. A. (2012). Volatile characterization by multivariate optimization of headspace-solid phase microextraction and sensorial evaluation of Chardonnay base wines. Journal of The Brazilian Chemical Society, 23, 678-687.

Welke, J. E., \& Zini, C. A. (2011). Comprehensive two-dimensional gas chromatography for analysis of volatile compounds in foods and beverages. Journal of The Brazilian Chemical Society, 22, 609-622. 
Werkhoff, P., Guntert, M., Krammer, G., Sommer, H., \& Kaulen, J. (1998). Vacuum headspace method in aroma research: flavor chemistry of yellow passion fruits. Journa of Agricultural and Food Chemistry, 46, 1076-1093.

Whetstine, M. E. C., Karagul-Yuceer, Y., Avsar, Y. K., \& Drake, M. A. (2003). Identification and quantification of character aroma components in fresh chevre-style goat cheese. Journal of Food Science, 68, 2441-2447.

Wu, S. M., Krings, U., Zorn, H., \& Berger, R. G. (2005). Volatile compounds from the fruiting bodies of beefsteak fungus Fistulina hepatica (Schaeffer: Fr.) Fr. Food Chemistry, 92, 221-226.
Zhao, Y., Xu, Y., Li, J., Fan, W., \& Jiang, W. (2009). Profile of volatile compounds in 11 brandies by headspace solid-phase microextraction followed by gas chromatography-mass spectrometry. Journal of Food Science, 74, C90-C99.

Zheng, L. Y., Sun, G. M., Liu, Y. G., Lv, L. L., Yang, W. X., Zhao, W. F., et al. (2012). Aroma volatile compounds from two fresh pineapple varieties in China. International Journal of Molecular Sciences, 13, 7383-7392. 